\title{
Stratified periodic water waves with singular density gradients
}

\author{
Joachim Escher ${ }^{1} \cdot$ Patrik Knopf $^{2} \cdot$ Christina Lienstromberg $^{3} \cdot$ Bogdan-Vasile Matioc $^{2}$ (D
}

Received: 3 December 2019 / Accepted: 23 January 2020 / Published online: 8 February 2020

(c) The Author(s) 2020

\begin{abstract}
We consider Euler's equations for free surface waves traveling on a body of density stratified water in the scenario when gravity and surface tension act as restoring forces. The flow is continuously stratified, and the water layer is bounded from below by an impermeable horizontal bed. For this problem we establish three equivalent classical formulations in a suitable setting of strong solutions which may describe nevertheless waves with singular density gradients. Based upon this equivalence we then construct twodimensional symmetric periodic traveling waves that are monotone between each crest and trough. Our analysis uses, to a large extent, the availability of a weak formulation of the water wave problem, the regularity properties of the corresponding weak solutions, and methods from nonlinear functional analysis.
\end{abstract}

Keywords Euler equations · Traveling waves $\cdot$ Stratified fluid $\cdot$ Singular density gradient

Mathematics Subject Classification $35 \mathrm{Q} 35 \cdot 35 \mathrm{~B} 32 \cdot 76 \mathrm{~B} 47 \cdot 76 \mathrm{~B} 70$

Bogdan-Vasile Matioc

bogdan.matioc@ur.de

Joachim Escher

escher@ifam.uni-hannover.de

Patrik Knopf

patrik.knopf@ur.de

Christina Lienstromberg

lienstromberg@iam.uni-bonn.de

1 Institut für Angewandte Mathematik, Leibniz Universität Hannover, Welfengarten 1, 30167 Hannover, Germany

2 Fakultät für Mathematik, Universität Regensburg, 93053 Regensburg, Germany

3 Institut für Angewandte Mathematik, Universität Bonn, Endenicher Allee 60, 53115 Bonn, Germany 


\section{Introduction}

Stratification is a phenomenon that is common in ocean flows where in the presence of salinity and under the influence of the gravitational force a heterogeneity in the fluid is produced. Stratification corresponds to the formation of fluid layers, normally arranged horizontally with the less dense layers being located on top of the denser ones. This phenomenon may be caused by many other factors including temperature, pressure, topography and oxygenation. Because of the plethora of effects resulting from stratification, such flows have received much attention, especially in geophysical fluid dynamics. In the setting of traveling stratified waves the problem is modeled by the stationary Euler equations for incompressible fluids, subject to natural boundary conditions, cf. (2.2). The study of two-dimensional stratified flows dates back to the pioneering work of Dubreil-Jacotin. In 1937 Dubreil-Jacotin [23] constructed small-amplitude stratified traveling gravity waves by using power series expansions. Previously in [22] she showed that Gerstner's explicit solution [10, 27] can be accommodated to describe exact traveling gravity waves with an arbitrary stratification. Furthermore, related to Gerstner's solution, there is a further exact solution describing an edge wave propagating along a sloping beach $[9,43,48]$ allowing even for an arbitrary stratification. Recently also other exact and explicit solutions of stratified flows in different geophysical regimes have been found, cf. [12, 16, 17, 29-31, 36].

Many of the papers dedicated to the stratified water wave problem consider the vertical stratification to be fairly smooth. Small-amplitude periodic gravity water waves possessing a linear stratification have been constructed in [25]. These waves may contain critical layers and stagnation points and the authors in [25] provide also the qualitative picture of the flow beneath the constructed waves. Small-amplitude periodic capillary-gravity waves with sufficiently regular density which may still contain critical layers have been found in [34] by means of local bifurcation. The local bifurcation branches of solutions to the stratified water wave problem have been extended by using global bifurcation theory to global branches in [32]. The papers [25, 32, 34] use the Long-Yih formulation [37, 56] (see (2.6)) of the problem whose availability is facilitated by the fact that the density is sufficiently regular. When excluding critical layers and stagnation points the stratified wave problem can be considered by using Dubreil-Jacotin's formulation (see (2.10)-(2.11)). This approach has been followed in [52-54] where-by means of local and global bifurcation theory-small- and large-amplitude stratified periodic water waves of finite depth are constructed both in the presence and absence of surface tension. The existence of solitary free surface water waves with general regular density distribution, together with a qualitative study of such flows, has been provided only recently [8], using again Dubreil-Jacotin's formulation in their treatise. Qualitative properties of stratified water waves with regular density, such as symmetry, regularity, and the unique determination of the wave when knowing the pressure on the bed and the fluid stratification, have been addressed in [7, 33, 51, 55].

In ocean flows, however, the density varies strongly in thin layers called pycnoclines which exhibit sharp density gradients, cf., e.g., [21, 46, 47]. For this reason some of the research $[3,4,14,15,21,39,40,42,49]$ is restricted to so-called layered models which consider the flow as consisting of a finite number of vertical layers each of them having uniform density. These layers are separated by internal waves which are mainly driven by the density difference between the layers (some models also consider surface tension effects). In this paper we consider a general continuous stratification, but allow for solutions with a density gradient which is merely $L_{r}$-integrable with $r \in(1, \infty)$ arbitrarily close to 1 . Furthermore, Bernoulli's function, called vorticity function in the constant density case, is also a general 
function and is assumed to be $L_{r}$-integrable too. A similar setting has been studied in [6] but in the absence of surface tension forces. The authors of [6] deal with a layered model with the density in each layer varying continuously in such a way that the density gradient is $L_{r}$-integrable, but with $r>2$. The choice $r>2$ is related to the Sobolev embedding $W_{r}^{1}\left(\mathbb{R}^{2}\right) \hookrightarrow C^{1-2 / r}\left(\mathbb{R}^{2}\right)$. In this regime the different formulations of the water wave problem mentioned above are equivalent in the setting of periodic Sobolev solutions. Our first main result is an equivalence result for the three formulations of the problem in a suitable setting of strong solutions, cf. Theorem 2.1 (see Theorem 2.5 for the case when surface tension is neglected). The equivalence in these theorems holds for $r \in[1, \infty)$. For $r \in[1,2]$ the Sobolev regularity is too weak for the equations to be realized in $L_{r}$-spaces, and therefore our notion of strong solutions involves some complementary Hölder regularity. Here the Hölder exponent $\alpha=1-1 / r \in[0, \infty)$ results from the embedding $W_{r}^{1}(\mathbb{R}) \hookrightarrow C^{1-1 / r}(\mathbb{R})$. The main result Theorem 2.3, which relies on the equivalence in Theorem 2.1, establishes the existence of infinitely many periodic solutions to the stratified water wave problem having merely a $L_{r}$-integrable density gradient. Moreover, the wave profiles are symmetric with respect to crest and trough lines and strictly monotone in between them. The proof of Theorem 2.3 uses the Crandall-Rabinowitz theorem [20, Theorem 1.7] on bifurcation from simple eigenvalues in the context of a weak interpretation of Dubreil-Jacotin's formulation. For traveling water waves this idea was first used by Constantin and Strauss 19] to construct homogeneous periodic gravity water waves with discontinuous vorticity. The situation of heterogeneous water waves is slightly different as the equations in the bulk cannot be recast in divergence form, see also [6]. Due to the presence of surface tension we need to deal with a second-order nonlinear equation on the surface boundary corresponding to the dynamic boundary condition at the waves surface. Here we use a recent trick employed first in [41, 44] (a similar idea appears also in [3, 32, 45]) to transform this equation into a Dirichlet boundary condition perturbed by a nonlinear and nonlocal part of order -1 . A particular feature of our analysis is that we fix both the fluid bed and the mean depth of the fluid (within a period). This fact in combination with the weak regularity of the density gradient reduces the number of possible bifurcation parameters. For this reason the best (probably only) choice for a bifurcation parameter is the wavelength $\lambda$ (the wavelength has also been used in [24] as one of the bifurcation parameters). It is worth pointing out that this choice provides a remarkable identity in (see (4.32)) that leads us to a very simple and elegant dispersion relation, cf. Lemma 4.9.

The paper is organized as follows: In Sect. 2 we introduce the three formulations of the problem and we establish their equivalence in Theorems 2.1 (and Theorem 2.5). Moreover, we state our main result in Theorem 2.3 on the existence of laminar and nonlaminar flow solutions and some qualitative properties. In Sect. 3 we first introduce the notion of a weak solution to Dubreil-Jacotin's formulation and establish, by means of a shooting method, the existence of at least one laminar flow solution to this latter formulation. This solution does not depend on the horizontal variable, having thus parallel and flat streamlines, and it solves the problem for each value of $\lambda$. This set of laminar solutions (we have a solution for each $\lambda>0$ ) is then seen as the trivial branch of solutions to the problem. Merely the existence of the laminar solution imposes some restriction on the physical properties of the flows, cf. (3.10) and Example 3.4. In Sect. 4 we reformulate the equations as an abstract bifurcation problem and identify, by using methods from nonlinear functional analysis, a particular value $\lambda_{*}$ of the wavelength parameter where a local branch of nonlaminar weak solutions arises from the set of laminar solutions. For this we need to impose a further, quite explicit restriction in (4.12). The proof of Theorem 2.3 is then completed by showing that the weak solutions that were found 
are in fact strong solutions, cf. Proposition 4.17. This gain of regularity relies on the regularity result in Theorem 4.14, which is inspired by ideas presented in [13] and [26].

\section{Mathematical formulations and the main results}

We now present three classical formulations of the steady water wave problem for stratified fluids. We start with the classical Euler formulation. The motion of an inviscid, incompressible, and stratified fluid is described by the Euler equations

$$
\left\{\begin{array}{l}
\rho\left(u_{t}+u u_{x}+v u_{y}\right)=-P_{x} \\
\rho\left(v_{t}+u v_{x}+v v_{y}\right)=-P_{y}-g \rho \\
\rho_{t}+\rho_{x} u+\rho_{y} v=0 \\
u_{x}+v_{y}=0,
\end{array}\right.
$$

where $\rho$ is the fluid's density, $u$ is the horizontal velocity, $v$ is the vertical velocity, $P$ is the pressure, and $g$ is the gravitational acceleration. The fluid domain is bounded from below by the impermeable flat bed $y=-d$, where $d$ is a fixed positive constant, and $y=\eta(t, x)$ denotes the wave surface. In addition to the conservation of momentum which is expressed by the first two equations of the system, the fluid is assumed to be incompressible and mass conserving (these properties correspond to the third and fourth equations, respectively). Our analysis is restricted to the physically relevant case of positive density, that is we assume throughout this paper that there exists a constant $\rho_{0}>0$ such that

$$
\rho \geq \rho_{0} .
$$

The equations in the fluid domain are subject to the following boundary conditions

$$
\begin{cases}P=-\frac{\sigma \eta_{x x}}{\left(1+\eta_{x}^{2}\right)^{3 / 2}} & \text { on } y=\eta(t, x), \\ v=\eta_{t}+u \eta_{x} & \text { on } y=\eta(t, x), \\ v=0 & \text { on } y=-d,\end{cases}
$$

where the atmospheric pressure is set to zero and $\sigma \geq 0$ denotes the surface tension coefficient. As we are interested in periodic waves we introduce the positive constant $\lambda$ to denote the associated (minimal) wavelength. Moreover, we require that

$$
\int_{0}^{\lambda} \eta(t, x) \mathrm{d} x=0
$$

at each time $t$. This condition implies in particular that also the mean depth of the fluid is fixed. Traveling periodic waves correspond to solutions of the previously introduced equations that exhibit a $(t, x)$-dependence of the form

$$
(u, v, P, \rho)(t, x, y)=(u, v, P, \rho)(x-c t, y) \quad \text { and } \quad \eta(t, x)=\eta(x-c t),
$$

where $c>0$ is the wave speed, and which are $\lambda$-periodic in $x$. Observed from a frame that moves with the wave speed $c$, traveling waves appear to be steady and we are left with the free boundary value problem 


$$
\begin{cases}\rho(u-c) u_{x}+\rho v u_{y}=-P_{x} & \text { in } \Omega_{\eta}, \\ \rho(u-c) v_{x}+\rho v v_{y}=-P_{y}-g \rho & \text { in } \Omega_{\eta}, \\ (u-c) \rho_{x}+v \rho_{y}=0 & \text { in } \Omega_{\eta}, \\ u_{x}+v_{y}=0 & \text { in } \Omega_{\eta}, \\ P=-\frac{\sigma \eta^{\prime \prime}}{\left(1+\eta^{\prime 2}\right)^{3 / 2}} & \text { on } y=\eta(x), \\ v=(u-c) \eta^{\prime} & \text { on } y=\eta(x), \\ v=0 & \text { on } y=-d, \\ \int_{0}^{\lambda} \eta(x) \mathrm{d} x=0, & \end{cases}
$$

where

$$
\Omega_{\eta}:=\{(x, y): x \in \mathbb{R},-d<y<\eta(x)\} .
$$

We point out that since $u$ and $c$ appear only in terms of the difference $u-c$ in (2.2), we may view the quintuplet $(u-c, v, P, \rho, \eta)$ as being the unknown, each of these functions additionally being $\lambda$-periodic with respect to the horizontal variable $x$.

In order to study problem (2.2) analytically it is useful to consider equivalent formulations. To this end we define the so-called stream function $\psi$ by the relations

$$
\nabla \psi:=(-\sqrt{\rho} v, \sqrt{\rho}(u-c)) \text { in } \Omega_{\eta} \text { and } \psi=0 \text { on } y=\eta(x) .
$$

One may observe that in the moving frame the streamlines of the flow coincide with the level curves of the stream function. Moreover, the density $\rho$ and the total hydraulic head

$$
E:=P+\rho \frac{(u-c)^{2}+v^{2}}{2}+g \rho y \quad \text { in } \Omega_{\eta}
$$

are both constant along the streamlines. In particular, if we require that

$$
\sup _{\Omega_{\eta}}(u-c)<0,
$$

a condition which is a priori satisfied for homogeneous irrotational water waves, the hodograph transformation $\mathcal{H}: \bar{\Omega}_{\eta} \rightarrow \bar{\Omega}$ defined by

$$
\mathcal{H}(x, y):=(q(x, y), p(x, y)):=(x,-\psi(x, y))
$$

is a bijection. Here $\Omega:=\mathbb{R} \times\left(p_{0}, 0\right)$ and $p_{0}:=-\left.\psi\right|_{y=-d}$ is a negative constant. Using this property one can find two functions $\bar{\rho}, \beta:\left[p_{0}, 0\right] \rightarrow \mathbb{R}$, the so-called streamline density function and the Bernoulli function, respectively, such that

$$
\rho \circ \mathcal{H}^{-1}=\bar{\rho} \quad \text { and } \quad-\partial_{p}\left(E \circ \mathcal{H}^{-1}\right)=\beta .
$$

In particular $\rho(x, y)=\bar{\rho}(-\psi(x, y))$ in $\Omega_{\eta}$. As the density usually increases with depth we restrict our considerations to the stably stratified regime defined by the inequality ${ }^{1}$

$$
\bar{\rho}^{\prime} \leq 0
$$

\footnotetext{
1 The assumption that the density is nondecreasing with depth is not needed for the equivalence result in Theorem 2.1 (or Theorem 2.5), but is used to a large extent in the proof of the bifurcation result in Theorem 2.3.
} 
These considerations lead one to the Long-Yih $[37,56]$ formulation of the hydrodynamical problem (2.2):

$$
\begin{cases}\Delta \psi=g y \bar{\rho}^{\prime}(-\psi)+\beta(-\psi) & \text { in } \Omega_{\eta}, \\ \psi=0 & \text { on } y=\eta(x), \\ \psi=-p_{0} & \text { on } y=-d, \\ |\nabla \psi|^{2}-\frac{2 \sigma \eta^{\prime \prime}}{\left(1+\eta^{\prime 2}\right)^{3 / 2}}+2 g \bar{\rho}(0) y=Q & \text { on } y=\eta(x) .\end{cases}
$$

Under the assumption (2.1) of positive density the condition (2.3) is equivalent to

$$
\sup _{\Omega_{\eta}} \psi_{y}<0
$$

The constant $Q$ in Eq. (2.6) $)_{4}$ is related to the energy $E$. Equation (2.6) $)_{4}$ identifies $Q$ for waves with zero integral mean as follows:

$$
Q=\frac{1}{\lambda} \int_{0}^{\lambda}|\nabla \psi|^{2}(x, \eta(x)) \mathrm{d} x .
$$

Using the (partial) hodograph transformation $\mathcal{H}$, a further equivalent formulation of (2.2)-(2.3) may be derived in terms of the height function $h: \bar{\Omega} \rightarrow \mathbb{R}$ that is defined by

$$
h(q, p)=y+d \quad \text { for }(q, p) \in \bar{\Omega} .
$$

The system (2.6) can then be recast in the fixed rectangular domain $\Omega$ in the following form:

$$
\begin{cases}\left(1+h_{q}^{2}\right) h_{p p}-2 h_{q} h_{p} h_{p q}+h_{p}^{2} h_{q q}-\left[g \bar{\rho}^{\prime}(h-d)+\beta\right] h_{p}^{3}=0 & \text { in } \Omega, \\ h=0 & \text { on } p=p_{0}, \\ 1+h_{q}^{2}+h_{p}^{2}\left[2 g \bar{\rho}(0)(h-d)-\frac{2 \sigma h_{q q}}{\left(1+h_{q}^{2}\right)^{3 / 2}}-\frac{1}{\lambda} \int_{0}^{\lambda} \frac{1+h_{q}^{2}}{h_{p}^{2}}(q, 0) \mathrm{d} q\right]=0 & \text { on } p=0,\end{cases}
$$

the relation (2.3) taking the form

$$
\inf _{\Omega} h_{p}>0 .
$$

In virtue of (2.11) the quasilinear equation $(2.10)_{1}$ is uniformly elliptic. This equation is complemented by a nonlinear and nonlocal boundary condition on $p=0$ and a homogeneous Dirichlet condition on $p=p_{0}$. This formulation gives an insight into the flow as the streamlines in the moving frame are parameterized by the mappings $[x \mapsto h(x, p)-d]$. In the setting of classical solutions it is not difficult to show that the three formulations (2.2)-(2.3), (2.6)-(2.8), and (2.10)-(2.11) are equivalent, cf., e.g., [11, 52-54]. This feature remains true in the more general framework described below.

Equivalent formulations In Theorem 2.1 we present our first main result which establishes for capillary-gravity stratified water waves, that is for $\sigma>0$, the equivalence of the three formulations in a suitable setting of strong solutions. The case $\sigma=0$ is treated in Theorem 2.5. A strong solution of any of the three formulations possesses weak derivatives up to highest order (the order is required by the equations) that are $L_{r}$-integrable. Moreover the lower order derivatives enjoy some additional Hölder regularity to ensure that all equations are satisfied in $L_{r}$-spaces (in particular pointwise a.e.). 
Theorem 2.1 (Equivalence for $\sigma>0$ ) Let $\sigma, \lambda>0$, and assume that (2.1) holds true. Given $r \in[1, \infty)$, set $\alpha:=(r-1) / r \in[0,1)$. Then, the following formulations are equivalent:

(i) The velocity formulation (2.2)-(2.3) for $u-c, v, P \in W_{r}^{1}\left(\Omega_{\eta}\right) \cap \mathrm{C}^{\alpha}\left(\bar{\Omega}_{\eta}\right), \eta \in W_{r}^{2}(\mathbb{R})$, and $\rho \in W_{r}^{1}\left(\Omega_{\eta}\right) \cap \mathrm{C}^{\alpha}\left(\bar{\Omega}_{\eta}\right)$.

(ii) The stream function formulation (2.6)-(2.8) for $\psi \in W_{r}^{2}\left(\Omega_{\eta}\right) \cap C^{1+\alpha}\left(\bar{\Omega}_{\eta}\right), \eta \in W_{r}^{2}(\mathbb{R})$, $\bar{\rho} \in W_{r}^{1}\left(\left(p_{0}, 0\right)\right)$, and $\beta \in L_{r}\left(\left(p_{0}, 0\right)\right)$.

(iii) The height function formulation (2.10)-(2.11) for $h \in W_{r}^{2}(\Omega) \cap \mathrm{C}^{1+\alpha}(\bar{\Omega})$ with $\operatorname{tr}_{0} h \in W_{r}^{2}(\mathbb{R}), \bar{\rho} \in W_{r}^{1}\left(\left(p_{0}, 0\right)\right)$, and $\beta \in L_{r}\left(\left(p_{0}, 0\right)\right)$.

The proof of Theorem 2.1 and the corresponding result for $\sigma=0$ are presented at the end of this section. It is worthwhile to add the following remarks.

\section{Remark 2.2}

(a) Given $r \geq 1$, the Hölder coefficient $\alpha:=(r-1) / r \in[0,1)$ corresponds to the onedimensional Sobolev embedding $W_{r}^{1}(\mathbb{R}) \hookrightarrow \mathrm{C}^{\alpha}(\mathbb{R})$. It is worthwhile to note that $W_{r}^{1}\left(\mathbb{R}^{2}\right)$ is not embedded in a space of continuous functions if $r \in[1,2]$. Therefore, the Hölder regularity required above is not implied by the Sobolev regularity.

(b) All function spaces in Theorem 2.1 consist only of functions that are $\lambda$-periodic with respect to $x$ and $q$, respectively.

(c) The symbol $\operatorname{tr}_{0}$ stands for the trace operator with respect to the boundary component $p=0$ of $\Omega=\mathbb{R} \times\left(p_{0}, 0\right)$, that is $\operatorname{tr}_{0} h(q)=h(q, 0), q \in \mathbb{R}$, for $h \in \mathrm{C}(\bar{\Omega})$.

(d) Let $\Omega \subset \mathbb{R}^{n}$ with $n \geq 1$ be open. In the proof of Theorem 2.1 (and also later on) we make use of the following properties

- $\partial(u v)=u \partial v+v \partial u$ in $\mathcal{D}^{\prime}(\Omega)$ for $u, v \in W_{1, l o c}^{1}(\Omega)$ with $u v, u \partial v+v \partial u \in L_{1, l o c}(\Omega)$;

- $W_{r}^{1}(\Omega) \cap \mathrm{BC}^{\alpha}(\Omega)$ is an algebra;

- If $f \in W_{1, l o c}^{1}(\Omega) \cap \mathrm{BC}(\Omega)$ has weak derivatives $f_{i} \in \mathrm{BC}(\Omega), 1 \leq i \leq n$, then $f \in \mathrm{BC}^{1}(\Omega)$.

The properties (2.12) and (2.14) are classical results, while (2.13) is a direct consequence of (2.12).

Local bifurcation The main issue of this paper is the local bifurcation result stated below. Under the natural assumptions (2.1) and (2.5) on the fluid density and the following restrictions on the physical quantities ${ }^{2}$

\footnotetext{
${ }^{2}$ The relations (2.15) are satisfied for example if $d$ is small compared to $\left|p_{0}\right|$. Furthermore, if $\beta=0=\bar{\rho}$, then $\mu_{*}=0$ and the first condition in (2.15) is trivially satisfied.
} 


$$
d+\frac{p_{0}}{\left(\mu_{*}-2 \min _{\left[p_{0}, 0\right]} B\right)^{1 / 2}}<0 \text { and } \frac{g d^{3} \bar{\rho}\left(p_{0}\right)\left|p_{0}\right|}{\left[p_{0}^{2}-\left(\mu_{*}-2 \min _{\left[p_{0}, 0\right]} B\right) d^{2}\right]^{3 / 2}} \leq \frac{x_{*}}{2},
$$

where $x_{*} \approx 1.9368$ is the positive solution to $e^{x}-x=5$,

$$
\mu_{*}:=2\left(g d\left\|\bar{\rho}^{\prime}\right\|_{L_{1}\left(\left(p_{0}, 0\right)\right)}+\max _{\left[p_{0}, 0\right]} B\right), \quad \text { and } \quad B(p):=\int_{p_{0}}^{p} \beta(s) \mathrm{d} s, \quad p \in\left[p_{0}, 0\right],
$$

we prove that the water wave problem (2.2)-(2.3) possesses, for each $\lambda>0$, at least one laminar flow solution with flat streamlines. Besides, a critical wavelength $\lambda_{*}>0$ is identified such that (2.2)-(2.3) has also other solutions with nonflat wave surface and with wavelength close to $\lambda_{*}$. More precisely, the following result holds true.

Theorem 2.3 Let $\sigma, d,-p_{0} \in(0, \infty), r \in(1, \infty)$, and $\alpha:=(r-1) / r \in(0,1)$ be given. Assume further that $\bar{\rho} \in W_{r}^{1}\left(\left(p_{0}, 0\right)\right)$ and $\beta \in L_{r}\left(\left(p_{0}, 0\right)\right)$ satisfy (2.1), (2.5), and (2.15). Then there exists a local bifurcation curve

$$
\mathcal{C}=\{(\lambda(s), u(s)-c, v(s), P(s), \rho(s), \eta(s)): s \in(-\varepsilon, \varepsilon)\},
$$

where $\varepsilon>0$ is small, having the following properties:

(i) $\lambda$ is smooth, $\lambda(s)>0$ for all $s>0$, and

$$
\lambda(s)=\lambda_{*}+O(s) \text { for } s \rightarrow 0,
$$

where $\lambda_{*}>0$ is defined in Proposition 4.11.

(ii) $(u(0)-c, v(0), P(0), \rho(0), \eta(0))$ is a strong solution to (2.2)-(2.3) for each $\lambda>0$, has flat streamlines, streamline density $\bar{\rho}$, and Bernoulli function $\beta$.

(iii) Given $s \in(-\varepsilon, \varepsilon) \backslash\{0\},(u(s)-c, v(s), P(s), \rho(s), \eta(s))$ is a strong solution to (2.2)(2.3) with minimal period $\lambda(s)$, streamline density $\bar{\rho}$, and Bernoulli function $\beta$. Moreover, the wave profile has precisely one crest and one trough per period, is symmetric with respect to crest and trough lines, and is strictly monotone between crest and trough.

(iv) The wave profile and all other streamlines are real-analytic graphs.

\section{Remark 2.4}

(a) We point out that we do not impose any restrictions on the value of $\sigma>0$, cf. (2.15). Nevertheless, the critical wavelength $\lambda_{*}$ depends in an intricate way on $\sigma$.

(b) The regularity of the parameterization of $\mathcal{C}$ and the asymptotic behavior of $\eta(s)$ as $s \rightarrow 0$ are specified in the proof of Theorem 2.3 at the end of Sect. 4.

(c) The strong solution $(u(0)-c, v(0), P(0), \rho(0), \eta(0))$ to (2.2)-(2.3) found in (ii) is called laminar flow solution. Its existence is established in Proposition 3.5.

(d) The limiting case $r=1$ remains open in the context of Theorems 2.3 and 4.14.

We conclude this section by proving the equivalence of the three formulations in the setting of strong solutions introduced above. 
Proof of Theorem 2.1 We start with the implication (i) $\Longrightarrow$ (ii). Let $(u-c, v, P, \rho, \eta)$ be a solution to (2.2)-(2.3). In virtue of (2.1) and the weak chain rule [28, Lemma 7.5] it follows that $\sqrt{\rho} \in W_{r}^{1}\left(\Omega_{\eta}\right) \cap \mathrm{C}^{\alpha}\left(\bar{\Omega}_{\eta}\right)$. Relation (2.13) then yields

$$
U:=\sqrt{\rho}(u-c), V:=\sqrt{\rho} v \in W_{r}^{1}\left(\Omega_{\eta}\right) \cap C^{\alpha}\left(\bar{\Omega}_{\eta}\right) .
$$

We note that the relations $(2.2)_{3}-(2.2)_{4}$ imply

$$
U_{x}+V_{y}=0 \text { in } L_{r}\left(\Omega_{\eta}\right) .
$$

For $(x, y) \in \bar{\Omega}_{\eta}$ we now define

$$
\psi(x, y):=-p_{0}+\int_{-d}^{y} U(x, s) \mathrm{d} s,
$$

where $p_{0}<0$ is a constant to be fixed below. It is obvious that $\psi$ is continuously differentiable with respect to $y$ with $\psi_{y}=U$. Moreover, making use of Fubini's theorem, the generalized Gauß theorem in [1, Appendix A 8.8], and the relations (2.17) and (2.2) ${ }_{7}$, we find for $\xi \in \mathrm{C}_{0}^{\infty}\left(\Omega_{\eta}\right)$ that

$$
\begin{aligned}
\int_{\Omega_{\eta}} \psi(x, y) \xi_{x}(x, y) \mathrm{d}(x, y) & =\int_{\Omega_{\eta}} U(x, s)\left(\int_{s}^{\eta(x)} \xi_{x}(x, y) \mathrm{d} y\right) \mathrm{d}(x, s)=\int_{\Omega_{\eta}} U(x, s) \phi_{x}(x, s) \mathrm{d}(x, s) \\
& =-\int_{\Omega_{\eta}} U_{x}(x, s) \phi(x, s) \mathrm{d}(x, s)=\int_{\Omega_{\eta}} V_{s}(x, s) \phi(x, s) \mathrm{d}(x, s) \\
& =-\int_{\Omega_{\eta}} V(x, s) \phi_{s}(x, s) \mathrm{d}(x, s)=\int_{\Omega_{\eta}} V(x, s) \xi(x, s) \mathrm{d}(x, s),
\end{aligned}
$$

where $\phi \in \mathrm{C}^{1}\left(\bar{\Omega}_{\eta}\right)$ is defined by the formula

$$
\phi(x, s):=\int_{s}^{\eta(x)} \xi(x, y) \mathrm{d} y, \quad(x, y) \in \bar{\Omega}_{\eta} .
$$

Thus, $\nabla \psi=(-V, U)$ and since these weak derivatives belong to $W_{r}^{1}\left(\Omega_{\eta}\right)$, we conclude that $\psi \in W_{r}^{2}\left(\Omega_{\eta}\right)$. Moreover, (2.14) implies that $\psi \in \mathrm{C}^{1+\alpha}\left(\bar{\Omega}_{\eta}\right)$.

The relation (2.7) is clearly satisfied in view of (2.3). Since $\psi$ is constant on the fluid bed and by $(2.2)_{6}$ also on the free surface $y=\eta(x)$, we infer from (2.7) that we may chose the negative constant $p_{0}$ such that $\psi=0$ on the free surface.

It is easy to see now that the mapping $\mathcal{H}$ defined in (2.4) satisfies $\mathcal{H} \in \operatorname{Diff}^{1+\alpha}\left(\Omega_{\eta}, \Omega\right)$, i.e., $\mathcal{H}: \Omega_{\eta} \rightarrow \Omega$ is a $C^{1+\alpha}$-diffeomorphism, with

$$
\left(\begin{array}{ll}
\frac{\partial q}{\partial x} & \frac{\partial q}{\partial y} \\
\frac{\partial p}{\partial x} & \frac{\partial p}{\partial y}
\end{array}\right)=\left(\begin{array}{ll}
1 & 0 \\
V & -U
\end{array}\right) \text { and }\left(\begin{array}{ll}
\frac{\partial x}{\partial q} & \frac{\partial x}{\partial p} \\
\frac{\partial y}{\partial q} & \frac{\partial y}{\partial p}
\end{array}\right) \circ \mathcal{H}=\left(\begin{array}{ll}
1 & 0 \\
\frac{V}{U} & -\frac{1}{U}
\end{array}\right) .
$$

In view of $\rho \in W_{r}^{1}\left(\Omega_{\eta}\right)$ it follows that $\rho \circ \mathcal{H}^{-1} \in W_{r}^{1}(\Omega)$ with

$$
\partial_{q}\left(\rho \circ \mathcal{H}^{-1}\right) \circ \mathcal{H}=\rho_{x}+\frac{V}{U} \rho_{y}=0,
$$

cf. $(2.2)_{3}$. Consequently, there exists $\bar{\rho} \in L_{r}\left(\left(p_{0}, 0\right)\right)$ with $\rho \circ \mathcal{H}^{-1}=\bar{\rho}$. Moreover, it actually holds that $\bar{\rho} \in W_{r}^{1}\left(\left(p_{0}, 0\right)\right)$ with weak derivative $\bar{\rho}^{\prime}=-\left(\rho_{y} / U\right) \circ \mathcal{H}^{-1}$. 
We now consider the expression

$$
E:=P+\frac{U^{2}+V^{2}}{2}+g \rho y
$$

which defines a function in $W_{r}^{1}\left(\Omega_{\eta}\right) \cap \mathrm{C}^{\alpha}\left(\bar{\Omega}_{\eta}\right)$. Hence, $E \circ \mathcal{H}^{-1} \in W_{r}^{1}(\Omega)$ and

$$
\begin{aligned}
\partial_{q}\left(E \circ \mathcal{H}^{-1}\right) \circ \mathcal{H}= & E_{x}+\frac{V}{U} E_{y} \\
= & \left(\rho(u-c) u_{x}+\rho v u_{y}+P_{x}\right)+\frac{V}{U}\left(\rho(u-c) v_{x}+\rho v v_{y}+P_{y}+g \rho\right) \\
& +\left((u-c) \rho_{x}+v \rho_{y}\right) \frac{E-P}{\sqrt{\rho} U} \quad \text { in } L_{r}\left(\Omega_{\eta}\right) .
\end{aligned}
$$

Appealing to $(2.2)_{1}-(2.2)_{3}$, it follows that $\partial_{q}\left(E \circ \mathcal{H}^{-1}\right)=0$. This relation has at least two implications. Firstly, $E$ is constant at the wave surface, which implies the existence of a constant $Q$ such that

$$
E=P+\frac{|\nabla \psi|^{2}}{2}+g \rho y=\frac{Q}{2} \quad \text { on } \quad y=\eta(x) .
$$

Secondly, in view of $\partial_{q}\left(\partial_{p}\left(E \circ \mathcal{H}^{-1}\right)\right)=0$, we may conclude that there exists a function $\beta \in L_{r}\left(\left(p_{0}, 0\right)\right)$ such that $-\partial_{p}\left(E \circ \mathcal{H}^{-1}\right)=\beta$. The relation (2.18) together with $(2.2)_{5}$ and $(2.2)_{8}$ shows that (2.6) ${ }_{4}$ holds true with $Q$ as defined in (2.8). Finally, since $\Delta \psi=U_{y}-V_{x}$, $(2.2)_{2}-(2.2)_{3}$ lead us to

$$
\beta \circ \mathcal{H}=\frac{1}{U} E_{y}=\Delta \psi-g y \bar{\rho}^{\prime} \circ \mathcal{H}
$$

which is the semilinear elliptic equation in (2.6). This completes this first step of the proof.

We now verify that (ii) $\Longrightarrow$ (iii). Let thus $(\psi, \eta)$ be a solution to (2.6)-(2.8) and let $h$ be the height function introduced in (2.9). Then, it follows that $h \in \mathrm{C}^{1+\alpha}(\bar{\Omega})$ with

$$
h_{q}=-\frac{\psi_{x}}{\psi_{y}} \circ \mathcal{H}^{-1} \quad \text { and } \quad h_{p}:=-\frac{1}{\psi_{y}} \circ \mathcal{H}^{-1}
$$

With regard to [28, Lemma 7.5], property (2.7) shows that $1 / \psi_{y} \in W_{r}^{1}\left(\Omega_{\eta}\right) \cap \mathrm{C}^{\alpha}\left(\bar{\Omega}_{\eta}\right)$, and the algebra property (2.13) leads us to the conclusion that $h_{q}, h_{p} \in W_{r}^{1}(\Omega)$, hence $h \in W_{r}^{2}(\Omega)$. The relation $(2.6)_{3}$ implies that $h$ satisfies $(2.10)_{2}$, while (2.11) follows immediately from (2.7). Moreover, since

$$
\psi_{x x}=\frac{h_{p}^{2} h_{q q}-2 h_{q} h_{p} h_{q p}+h_{q}^{2} h_{p p}}{h_{p}^{3}} \circ \mathcal{H} \quad \text { and } \quad \psi_{y y}=\frac{h_{p p}}{h_{p}^{3}} \circ \mathcal{H},
$$

it follows from (2.6) that $h$ is a solution to $(2.10)_{1}$. Let us also note that Eq. $(2.6)_{2}$ yields $\eta(q)=h(q, 0)-d, q \in \mathbb{R}$, and therefore $\operatorname{tr}_{0} h \in W_{r}^{2}(\mathbb{R})$. The boundary condition $(2.10)_{3}$ is a direct consequence of (2.6) 4 . This completes the second step of the proof.

It remains to establish the implication (iii) $\Longrightarrow$ (i). To begin we first define $\eta:=h(\cdot, 0)-d$. Then $\eta \in W_{r}^{2}(\mathbb{R})$ and integrating $(2.10)_{3}$ over one period of the wave we find that $(2.2)_{8}$ is satisfied. Besides, (2.11) yields that $\eta(x)+d=h(x, 0)>0$ for all $x \in \mathbb{R}$. We now associate with $\eta$ the corresponding velocity, pressure, and density distribution. To this end we let $\Phi: \mathbb{R}^{2} \times\left[2 p_{0}, 0\right] \rightarrow \mathbb{R}$ be the function defined by 


$$
\Phi(x, y, p):= \begin{cases}y+d-h(x, p), & p \in\left[p_{0}, 0\right] \\ y+d+h\left(x, 2 p_{0}-p\right), & p \in\left[2 p_{0}, p_{0}\right] .\end{cases}
$$

Then $\Phi \in C^{1+\alpha}\left(\mathbb{R}^{2} \times\left[0,2 p_{0}\right]\right), \Phi\left(x,-d, p_{0}\right)=0$ for all $x \in \mathbb{R}$, and $\Phi_{p} \leq-\inf _{\Omega} h_{p}<0$. For fixed, but arbitrary $x \in \mathbb{R}$, the implicit function theorem yields the existence of a function $\psi(x, \cdot)$ which is continuously differentiable in $[-d,-d+\varepsilon)$, for some $\varepsilon>0$, and satisfies $\psi(x,-d)=-p_{0}$ as well as

$$
h(x,-\psi(x, y))=y+d \text { for all } y \in[-d,-d+\varepsilon) .
$$

Because $\psi(x, \cdot)$ is strictly decreasing, we can extend this function continuously in $-d+\varepsilon$ if

$$
\lim _{y \rightarrow-d+\varepsilon} \psi(x, y)<0 .
$$

The implicit function theorem then enables us to even extend $\psi(x, \cdot)$ beyond $-d+\varepsilon$. Hence, $\psi(x, \cdot)$ has a maximal extension $\psi(x, \cdot) \in \mathrm{C}^{1}([-d, A(x)), \mathbb{R})$ with $\psi(x,-d)=-p_{0}$ and $\psi(x, A(x))=0$. In view of $A(x)+d=h(x, 0)=\eta(x)+d$, we conclude that $\eta(x)=A(x)$. Therefore $\psi: \bar{\Omega}_{\eta} \rightarrow \mathbb{R}$ and (2.7) is satisfied. Moreover, the implicit function theorem yields that $\psi \in \mathrm{C}^{1+\alpha}\left(\Omega_{\eta}\right)$ with

$$
\psi_{x}(x, y)=\frac{h_{q}(x,-\psi(x, y))}{h_{p}(x,-\psi(x, y))} \quad \text { and } \quad \psi_{y}(x, y)=-\frac{1}{h_{p}(x,-\psi(x, y))} .
$$

Since $h \in \mathrm{C}^{1+\alpha}(\bar{\Omega})$, it now follows that $\psi \in \mathrm{C}^{1+\alpha}\left(\bar{\Omega}_{\eta}\right)$ and the mapping $\mathcal{H}$ defined in (2.4) obviously satisfies $\mathcal{H} \in \operatorname{Diff}^{1+\alpha}\left(\Omega_{\eta}, \Omega\right)$. Since $h_{q}, h_{p} \in W_{r}^{1}(\Omega) \cap \mathrm{C}^{\alpha}(\Omega)$, we find in virtue of (2.12)-(2.13) and [28, Lemma 7.5] that $\psi \in W_{r}^{2}(\Omega)$. Moreover, the derivatives $\psi_{x x}$ and $\psi_{y y}$ satisfy (2.19). We now define $\rho, u-c, v: \bar{\Omega}_{\eta} \rightarrow \mathbb{R}$ by setting

$$
\rho=\bar{\rho} \circ \mathcal{H}, \quad \sqrt{\rho}(u-c)=\psi_{y}, \quad \sqrt{\rho} v=-\psi_{x},
$$

and we let $P: \bar{\Omega}_{\eta} \rightarrow \mathbb{R}$ be given by the relation

$$
P(x, y)=-\rho \frac{(u-c)^{2}+v^{2}}{2}(x, y)-g \rho(x, y) y-\int_{0}^{-\psi(x, y)} \beta(s) \mathrm{d} s+\frac{Q}{2},
$$

with $Q$ defined according to (2.8). Then $\rho \in W_{r}^{1}(\Omega) \cap C^{\alpha}(\bar{\Omega})$ and, recalling (2.1), we may argue as above to conclude that $u, v$, and $P$ belong to $W_{r}^{1}(\Omega) \cap C^{\alpha}(\bar{\Omega})$. It is now a matter of direct computation to see that all the equations of (2.2)-(2.3) are satisfied. This completes the proof.

It follows from the proof of Theorem 2.1 that, when neglecting surface tension effects, the following equivalence result holds.

Theorem 2.5 (Equivalence for $\sigma=0$ ) Let, $\sigma=0, \lambda>0$, and assume that (2.1) holds true. Given $r \in[1, \infty)$, set $\alpha:=(r-1) / r \in[0,1)$. Then, the following formulations are equivalent: 
(i) The velocity formulation (2.2)-(2.3) for $u-c, v, P \in W_{r}^{1}\left(\Omega_{\eta}\right) \cap \mathrm{C}^{\alpha}\left(\bar{\Omega}_{\eta}\right), \eta \in \mathrm{C}^{1+\alpha}(\mathbb{R})$, and $\rho \in W_{r}^{1}\left(\Omega_{\eta}\right) \cap C^{\alpha}\left(\bar{\Omega}_{\eta}\right)$.

(ii) The stream function formulation (2.6)-(2.8) for $\psi \in W_{r}^{2}\left(\Omega_{\eta}\right) \cap C^{1+\alpha}\left(\bar{\Omega}_{\eta}\right)$, $\eta \in \mathrm{C}^{1+\alpha}(\mathbb{R}), \bar{\rho} \in W_{r}^{1}\left(\left(p_{0}, 0\right)\right)$, and $\beta \in L_{r}\left(\left(p_{0}, 0\right)\right)$.

(iii) The height function formulation (2.10)-(2.11) for $h \in W_{r}^{2}(\Omega) \cap C^{1+\alpha}(\bar{\Omega})$, $\beta \in L_{r}\left(\left(p_{0}, 0\right)\right)$, and $\bar{\rho} \in W_{r}^{1}\left(\left(p_{0}, 0\right)\right)$.

\section{A weak setting for Dubreil-Jacotin's formulation}

In this section we seek solutions to problem (2.10)-(2.11) under the general assumptions that

$$
r \in(1, \infty), \quad \bar{\rho} \in W_{r}^{1}\left(\left(p_{0}, 0\right)\right), \quad \text { and } \quad \beta \in L_{r}\left(\left(p_{0}, 0\right)\right) \text {, }
$$

where $\bar{\rho}$ and $\beta$ are arbitrary but fixed. Moreover, we restrict to the setting of stably stratified flows defined by (2.1) and (2.5). The reason for studying the height function formulation is twofold. Firstly, the equations have a single unknown, the height function $h$, and secondly, the Bernoulli function $\beta$ and the streamline density $\bar{\rho}$ appear as coefficients in the equations.

Since we aim to formulate (2.10) as a bifurcation problem and to use the wavelength $\lambda$ as bifurcation parameter, we let

$$
\widetilde{h}(q, p):=h(\lambda q, p), \quad(q, p) \in \bar{\Omega} .
$$

Then $\widetilde{h}$ is 1-periodic ${ }^{3}$ and (2.10) may be rewritten (after dropping tildes) as

$$
\begin{cases}\left(\lambda^{2}+h_{q}^{2}\right) h_{p p}-2 h_{q} h_{p} h_{p q}+h_{p}^{2} h_{q q}-\lambda^{2}\left[g \bar{\rho}^{\prime}(h-d)+\beta\right] h_{p}^{3}=0 & \text { in } \Omega, \\ h=0 & \text { on } p=p_{0}, \\ \lambda^{2}+h_{q}^{2}+h_{p}^{2}\left[2 \lambda^{2} g \bar{\rho}(0)(h-d)-\frac{2 \sigma \lambda^{3} h_{q q}}{\left(\lambda^{2}+h_{q}^{2}\right)^{3 / 2}}-\int_{0}^{1} \frac{\lambda^{2}+h_{q}^{2}}{h_{p}^{2}}(q, 0) \mathrm{d} q\right]=0 & \text { on } p=0,\end{cases}
$$

while (2.11) remains unchanged. Now not only $h$ is unknown in (3.3) but also the wavelength $\lambda$.

In order to determine strong solutions to (3.3) and (2.11) as defined in Theorem 2.1(iii), we shall first find weak solutions to this problem and then improve their regularity. We now introduce a proper notion of weak solutions.

Definition 3.1 A function $h \in \mathrm{C}^{1}(\bar{\Omega})$ is called weak solution to (3.3) and (2.11) if $h$ satisfies (2.11), the equation ${ }^{4}$

\footnotetext{
${ }^{3}$ Hereinafter all function spaces consist of functions which are 1-periodic with respect to $q$ (provided that they depend on the variable $q$ ).

${ }^{4}$ Recall that $B$ denotes the primitive of the Bernoulli function, cf. (2.16).
} 


$$
\left(\frac{h_{q}}{h_{p}}\right)_{q}-\left(\frac{\lambda^{2}+h_{q}^{2}}{2 h_{p}^{2}}+\lambda^{2} B+\lambda^{2} g \bar{\rho}(h-d)\right)_{p}+\lambda^{2} g \bar{\rho} h_{p}=0 \quad \text { in } \mathcal{D}^{\prime}(\Omega),
$$

and the boundary conditions

$$
h=\left(1-\partial_{q}^{2}\right)^{-1} \operatorname{tr}_{0}\left[h-\frac{\left(\lambda^{2}+h_{q}^{2}\right)^{3 / 2}}{2 \sigma \lambda^{3}}\left(\frac{\lambda^{2}+h_{q}^{2}}{h_{p}^{2}}+2 \lambda^{2} g \bar{\rho}(h-d)-\int_{0}^{1} \frac{\lambda^{2}+h_{q}^{2}}{h_{p}^{2}} \mathrm{~d} q\right)\right] \quad \text { on } p=0
$$

and

$$
h=0 \quad \text { on } p=p_{0} .
$$

In Definition 3.1 we have made use of the fact that

$$
\left(1-\partial_{q}^{2}\right): \mathrm{C}^{2}(\mathbb{R}) \rightarrow \mathrm{C}(\mathbb{R})
$$

is an isomorphism.

Laminar flow solutions In the remainder of this section we show that, given any $\lambda>0$, Eqs. (3.3) and (2.11) have at least one weak solution $H$ that depends only on the variable $p$. This solution is then easily seen to be a strong solution to (3.3) and (2.11) (similar as defined in Theorem 2.1(iii)). This is the laminar flow solution mentioned in Theorem 2.3(ii).

Since the density is positive, it follows that $H=H(p)$ is a weak solution to (3.3) and (2.11) if and only if $H^{\prime}>0$ on $\left[p_{0}, 0\right]$ and if $H$ solves the system

$$
\left\{\begin{array}{l}
\left(\frac{1}{H^{2}}\right)^{\prime}=-2\left[g \bar{\rho}^{\prime}(H-d)+\beta\right] \quad \text { in } \mathcal{D}^{\prime}\left(\left(p_{0}, 0\right)\right), \\
H(0)=d \\
H\left(p_{0}\right)=0
\end{array}\right.
$$

We emphasize that the wavelength parameter does not appear in (3.4). Taking into account that $H \in \mathrm{C}^{1}\left(\left[p_{0}, 0\right]\right)$, we get that additionally $H \in W_{r}^{2}\left(\left(p_{0}, 0\right)\right)$. Moreover, setting $\mu:=\left(H^{\prime}\left(p_{0}\right)\right)^{-2}>0$, the function $H$ satisfies the fixed point equation

$$
H(p)=\int_{p_{0}}^{p}\left(\mu-2 \int_{p_{0}}^{r}\left[g \bar{\rho}^{\prime}(s)(H(s)-d)+\beta(s)\right] \mathrm{d} s\right)^{-1 / 2} d r, \quad p \in\left[p_{0}, 0\right] .
$$

Our goal is to show, by means of a shooting argument, that there exists a $\mu>0$ such that (3.5) has a solution which satisfies additionally $H(0)=d$. This solution then also solves (3.4). Let $\mu_{*} \geq 0$ and $B$ be as defined in (2.16). As a first step we prove below that the fixed point equation (3.5) has a unique nonnegative solution $H=H(\cdot ; \mu)$ for any $\mu>\mu_{*}$.

Proposition 3.2 Given $\mu>\mu_{*}$, there is a unique solution $H=H(\cdot ; \mu) \in W_{r}^{2}\left(\left(p_{0}, 0\right)\right)$ to the fixed point equation (3.5). It further holds that $H^{\prime}>0$ in $\left[p_{0}, 0\right]$.

Proof Let $\mu>\mu_{*}$ be fixed. Given $p_{1} \in\left(p_{0}, 0\right]$ and $H \in \mathrm{C}\left(\left[p_{0}, p_{1}\right],[0, \infty)\right)$, we define

$$
T_{1} H(p):=\int_{p_{0}}^{p}\left(\mu-2 \int_{p_{0}}^{r}\left[g \bar{\rho}^{\prime}(s)(H(s)-d)+\beta(s)\right] d s\right)^{-1 / 2} \mathrm{~d} r, \quad p \in\left[p_{0}, p_{1}\right] .
$$


We now show that $T_{1}$ is a self-map. Indeed, recalling that $\bar{\rho}^{\prime} \leq 0$, it holds that

$$
\begin{aligned}
& \mu-2 \int_{p_{0}}^{r}\left[g \bar{\rho}^{\prime}(s)(H(s)-d)+\beta(s)\right] \mathrm{d} s= \mu-2 B(r)-2 \int_{[H \leq d]} g \bar{\rho}^{\prime}(s)(H(s)-d) \mathrm{d} s \\
&-2 \int_{[H>d]} g \bar{\rho}^{\prime}(s)(H(s)-d) \mathrm{d} s \\
& \geq \mu-2 \max _{\left[p_{0}, 0\right]} B-2 \int_{p_{0}}^{p_{1}} g d\left|\bar{\rho}^{\prime}(s)\right| \mathrm{d} s \\
& \geq \mu-\mu_{*}>0, \quad r \in\left[p_{0}, p_{1}\right],
\end{aligned}
$$

and consequently $T_{1} H \in \mathrm{C}\left(\left[p_{0}, p_{1}\right],[0, \infty)\right)$.

If $p_{1}$ is sufficiently close to $p_{0}$, then $T_{1}$ is a contraction. Indeed, given $H, K \in \mathrm{C}\left(\left[p_{0}, p_{1}\right],[0, \infty)\right)$ and $p \in\left[p_{0}, p_{1}\right],(3.6)$ shows that

$$
\left|T_{1} H(p)-T_{1} K(p)\right| \leq \frac{g\left(p_{1}-p_{0}\right)\left\|\bar{\rho}^{\prime}\right\|_{L_{1}\left(\left(p_{0}, 0\right)\right)}}{\left(\mu-\mu_{*}\right)^{3 / 2}}\|H-K\|_{\mathrm{C}\left(\left[p_{0}, p_{1}\right]\right)} \leq \frac{1}{2}\|H-K\|_{\mathrm{C}\left(\left[p_{0}, p_{1}\right]\right)}
$$

if

$$
p_{1} \leq p_{1, \mu}:=\min \left\{0, p_{0}+\frac{\left(\mu-\mu_{*}\right)^{3 / 2}}{2 g\left(\left\|\bar{\rho}^{\prime}\right\|_{L_{1}\left(\left(p_{0}, 0\right)\right)}+1\right)}\right\} .
$$

Observing that $\mathrm{C}\left(\left[p_{0}, p_{1}\right],[0, \infty)\right)$ is a complete metric space, the Banach contraction principle yields the existence and uniqueness of a nonnegative solution $H_{1} \in W_{r}^{2}\left(\left(p_{0}, p_{1, \mu}\right)\right)$ to (3.5). We next prove that as long as the right endpoint of the interval of existence does not reach 0 , we may extend it to the right by the amount of

$$
\frac{\left(\mu-\mu_{*}\right)^{3 / 2}}{2 g\left(\left\|\bar{\rho}^{\prime}\right\|_{L_{1}\left(\left(p_{0}, 0\right)\right)}+1\right)} \text {. }
$$

Indeed, assume that $p_{1, \mu}<0$. Given $p_{2} \in\left(p_{1, \mu}, 0\right]$ and $H \in C\left(\left[p_{1, \mu}, p_{2}\right],[0, \infty)\right)$ we set

$$
T_{2} H(p):=H_{1}\left(p_{1, \mu}\right)+\int_{p_{1, \mu}}^{p}\left(c_{\mu}-2 \int_{p_{1, \mu}}^{r}\left[g \bar{\rho}^{\prime}(s)(H(s)-d)+\beta(s)\right] \mathrm{d} s\right)^{-1 / 2} \mathrm{~d} r
$$

for $p \in\left[p_{1, \mu}, p_{2}\right]$ and

$$
c_{\mu}:=\left(H_{1}^{\prime}\left(p_{1, \mu}\right)\right)^{-2}=\mu-2 \int_{p_{0}}^{p_{1, \mu}}\left[g \bar{\rho}^{\prime}(s)\left(H_{1}(s)-d\right)+\beta(s)\right] \mathrm{d} s>0 .
$$

The same arguments as above yield

$$
c_{\mu}-2 \int_{p_{1, \mu}}^{r}\left[g \bar{\rho}^{\prime}(s)(H(s)-d)+\beta(s)\right] \mathrm{d} s \geq \mu-\mu_{*}>0, \quad p \in\left[p_{1, \mu}, p_{2}\right],
$$

hence $\quad T_{2} H \in C\left(\left[p_{1, \mu}, p_{2}\right],[0, \infty)\right) \quad$ and $\quad\left(T_{2} H\right)^{(k)}\left(p_{1, \mu}\right)=H_{1}^{(k)}\left(p_{1, \mu}\right) \quad$ for $\quad k \in\{0,1\}$. Furthermore, given $H, K \in C\left(\left[p_{1, \mu}, p_{2}\right],[0, \infty)\right)$ and $p \in\left[p_{1, \mu}, p_{2}\right]$, it holds that 


$$
\left|T_{2} H(p)-T_{2} K(p)\right| \leq \frac{g\left(p_{2}-p_{1, \mu}\right)\left\|\bar{\rho}^{\prime}\right\|_{L_{1}\left(\left(p_{0}, 0\right)\right)}}{\left(\mu-\mu_{*}\right)^{3 / 2}}\|H-K\|_{C\left(\left[p_{1, \mu}, p_{2}\right]\right)} \leq \frac{1}{2}\|H-K\|_{C\left(\left[p_{1, \mu}, p_{2}\right]\right)}
$$

provided that

$$
p_{2} \leq p_{2, \mu}:=\min \left\{0, p_{1, \mu}+\frac{\left(\mu-\mu_{*}\right)^{3 / 2}}{2 g\left(\left\|\bar{\rho}^{\prime}\right\|_{\left.L_{1}\left(p_{0}, 0\right)\right)}+1\right)}\right\} \text {. }
$$

Hence, $T_{2}$ possesses a fixed point $H_{2} \in W_{r}^{2}\left(\left(p_{1, \mu}, p_{2, \mu}\right)\right)$. Thus, we may extend $H_{1}$ to a solution to (3.5) which lies in $W_{r}^{2}\left(\left(0, p_{2, \mu}\right)\right)$ and which equals $H_{2}$ on $\left(p_{1, \mu}, p_{2, \mu}\right)$. Arguing in this way, if necessary, we may extend (in a finite number of steps) $H_{1}$ onto the whole interval $\left[p_{0}, 0\right]$. The uniqueness claim is obvious.

We next show that the solution found in Proposition 3.2 depends smoothly on the parameter $\mu$.

Lemma 3.3 For any $\mu>\mu_{*}$, let $H(\cdot ; \mu)$ denote the solution to the fixed point equation (3.5) as given by Proposition 3.2. Then, the mapping

$$
[\mu \mapsto H(\cdot ; \mu)]:\left(\mu_{*}, \infty\right) \rightarrow C\left(\left[p_{0}, 0\right]\right)
$$

is smooth.

Proof We prove that, given $\varepsilon>0$, the mapping (3.8) is smooth on $\left(\mu_{*}+2 \varepsilon g\left\|\bar{\rho}^{\prime}\right\|_{L_{1}\left(\left(p_{0}, 0\right)\right)}, \infty\right)$. This claim follows by applying the implicit function theorem to the equation $\mathcal{F}(H, \mu)=0$, where $\mathcal{F}: \mathcal{U}_{\varepsilon} \times\left(\mu_{*}+2 \varepsilon g\left\|\bar{\rho}^{\prime}\right\|_{L_{1}\left(\left(p_{0}, 0\right)\right)}, \infty\right) \subset \mathrm{C}\left(\left[p_{0}, 0\right]\right) \times \mathbb{R} \rightarrow \mathrm{C}\left(\left[p_{0}, 0\right]\right)$ is defined by

$$
\mathcal{F}(H, \mu)(p):=H(p)-\int_{p_{0}}^{p}\left(\mu-2 \int_{p_{0}}^{r}\left[g \bar{\rho}^{\prime}(s)(H(s)-d)+\beta(s)\right] \mathrm{d} s\right)^{-1 / 2} \mathrm{~d} r, \quad p \in\left[p_{0}, 0\right] .
$$

Here

$$
\mathcal{U}_{\varepsilon}:=\left\{H \in \mathrm{C}\left(\left[p_{0}, 0\right]\right): H>-\varepsilon\right\}
$$

is an open subset of $\mathrm{C}\left(\left[p_{0}, 0\right]\right)$. Arguing as in the derivation of (3.6) it can be seen that the operator $\mathcal{F}$ is well-defined. Moreover, $\mathcal{F}$ is smooth. The partial derivative $\partial_{H} \mathcal{F}(H, \mu)[\widetilde{H}]$ of $\mathcal{F}$ with respect to $H$ at a given point $(H, \mu) \in \mathcal{U}_{\varepsilon} \times\left(\mu_{*}+2 \varepsilon g\left\|\bar{\rho}^{\prime}\right\|_{\left.L_{1}\left(p_{0}, 0\right)\right)}, \infty\right)$ can be expressed as

$$
\partial_{H} \mathcal{F}(H, \mu): \mathrm{C}\left(\left[p_{0}, 0\right]\right) \rightarrow \mathrm{C}\left(\left[p_{0}, 0\right]\right), \quad \widetilde{H} \mapsto \widetilde{H}-\mathcal{K}[\tilde{H}],
$$

where the operator $\mathcal{K}: \mathrm{C}\left(\left[p_{0}, 0\right]\right) \rightarrow \mathrm{C}\left(\left[p_{0}, 0\right]\right)$ is given by

$$
\mathcal{K}[\tilde{H}](p)=g \int_{p_{0}}^{p}\left(\mu-2 \int_{p_{0}}^{r}\left[g \bar{\rho}^{\prime}(H-d)+\beta\right] \mathrm{d} s\right)^{-3 / 2}\left(\int_{p_{0}}^{r} \bar{\rho}^{\prime} \widetilde{H} \mathrm{~d} s\right) \mathrm{d} r, \quad p \in\left[p_{0}, 0\right] .
$$

One can easily verify that $\mathcal{K}$ actually maps continuously into $W_{r}^{2}\left(\left(p_{0}, 0\right)\right)$. Since the embedding of $W_{r}^{2}\left(\left(p_{0}, 0\right)\right)$ into $\mathrm{C}\left(\left[p_{0}, 0\right]\right)$ is compact, it follows that $\mathcal{K}$ is a compact operator. Hence, $\partial_{H} \mathcal{F}(H, \mu)$ is a compact perturbation of the identity. Using the RieszSchauder theorem, we can conclude that $\partial_{H} \mathcal{F}(H, \mu)$ is a Fredholm operator of index zero. 
Furthermore, $\partial_{H} \mathcal{F}(H, \mu)[\tilde{H}]=0$ if and only if $\widetilde{H}=\mathcal{K}[\tilde{H}]$. In this case, $\widetilde{H}$ satisfies the inequality

$$
\begin{aligned}
|\widetilde{H}(p)| & \leq g \int_{p_{0}}^{p}\left(\mu-\mu_{*}-2 \varepsilon g\left\|\bar{\rho}^{\prime}\right\|_{L_{1}\left(\left(p_{0}, 0\right)\right)}\right)^{-3 / 2} \int_{p_{0}}^{r}\left|\bar{\rho}^{\prime}(s)\right| \cdot|\widetilde{H}(s)| \mathrm{d} s \mathrm{~d} r \\
& \leq g\left|p_{0}\right|\left(\mu-\mu_{*}-2 \varepsilon g\left\|\bar{\rho}^{\prime}\right\|_{L_{1}\left(\left(p_{0}, 0\right)\right)}\right)^{-3 / 2} \int_{p_{0}}^{p}\left|\bar{\rho}^{\prime}(s)\right| \cdot|\widetilde{H}(s)| \mathrm{d} s
\end{aligned}
$$

for all $p \in\left[p_{0}, 0\right]$. Hence, applying Gronwall's lemma, we obtain that $\widetilde{H}=0$ on $\left[p_{0}, 0\right]$. This means that $\operatorname{ker}\left(\partial_{H} \mathcal{F}(H, \mu)\right)=\{0\}$ and thus, according to Fredholm's alternative, $\partial_{H} \mathcal{F}(H, \mu)$ is an isomorphism from $\mathrm{C}\left(\left[p_{0}, 0\right]\right)$ to $\mathrm{C}\left(\left[p_{0}, 0\right]\right)$.

Due to the construction of $\mathcal{F}$ it holds that

$$
\mathcal{F}(H(\cdot ; \mu), \mu)=0 \quad \text { for all } \mu \in\left(\mu_{*}+2 \varepsilon g\left\|\bar{\rho}^{\prime}\right\|_{L_{1}\left(\left(p_{0}, 0\right)\right)}, \infty\right) .
$$

Since the solution to (3.5) is unique also in $\mathcal{U}_{\varepsilon}$ if $\mu>\mu_{*}+2 \varepsilon g\left\|\bar{\rho}^{\prime}\right\|_{L_{1}\left(\left(p_{0}, 0\right)\right)}$ (this follows in a similar way as in Proposition 3.2), the implicit function theorem implies that $[\mu \mapsto H(\cdot ; \mu)]$ is smooth in $\left(\mu_{*}+2 \varepsilon g\left\|\bar{\rho}^{\prime}\right\|_{L_{1}\left(\left(p_{0}, 0\right)\right)}, \infty\right)$ for all $\varepsilon>0$.

In order to establish the existence of a solution to (3.4) it remains to prove that there is a $\mu>\mu_{*}$ such that $H(0 ; \mu)=d$. To this end first note that (3.6) yields

$$
H(p ; \mu) \leq \frac{\left|p_{0}\right|}{\left(\mu-\mu_{*}\right)^{1 / 2}} \quad \text { for } p \in\left[p_{0}, 0\right]
$$

and hence $H(0 ; \mu)<d$ for sufficiently large $\mu$. If we additionally show that $H(0 ; \mu)>d$ for $\mu$ close to $\mu_{*}$, then the continuous dependence of $H$ on $\mu$ (Lemma 3.3) implies that $H(0 ; \mu)=d$ for some $\mu$. It turns out that this requires additional restrictions on the physical quantities. The following example illustrates the approach in the simplified case of constant density.

Example 3.4 Assume that $\bar{\rho}^{\prime}=0$ and that $\beta(p)=C\left(p-p_{0}\right)^{-1 / 2}$. Since the density is constant, the function $\beta$ has to be interpreted as the vorticity function of the flow, cf. [18]. Unbounded vorticity functions are of relevance for example for flows in channels [5]. By Proposition 3.2, Eq. (3.5) possesses a unique solution $H=H(p ; \mu)$ for each $\mu>\mu_{*}=2 C\left|p_{0}\right|^{1 / 2}$. The solution is given by the explicit formula

$$
H(p ; \mu)=\int_{p_{0}}^{p} \frac{1}{\sqrt{\mu-2 B(r)}} \mathrm{d} r, \quad p_{0} \leq p \leq 0 .
$$

Since $H(0 ; \mu)<H\left(0, \mu_{*}\right)$ for $\mu>\mu_{*}$ and because of

$$
H\left(0 ; \mu_{*}\right)=\int_{p_{0}}^{0} \frac{1}{\sqrt{2 B(0)-2 B(r)}} \mathrm{d} r=\frac{1}{2 \sqrt{C}} \int_{p_{0}}^{0} \frac{\sqrt{\left|p_{0}\right|^{1 / 2}+\left(r-p_{0}\right)^{1 / 2}}}{\sqrt{-r}} \mathrm{~d} r<\infty,
$$

the condition $H\left(0 ; \mu^{*}\right)>d$ has to be imposed, otherwise $H(0 ; \mu)<d$ for all $\mu>\mu_{*}$ and (3.4) has no solutions. 
Let us now return to the more involved setting of stratified waves addressed in this paper. Given $\mu>\mu_{*}$, we define

$$
p_{\mu}:=\min \left(\{0\} \cup H(\cdot ; \mu)^{-1}(\{d\})\right),
$$

Then recalling (3.9), we observe that $p_{\mu}=0$ and $H(0 ; \mu)<d$ if $\mu$ is sufficiently large. The size condition that we require reads

$$
d+\frac{p_{0}}{\left(\mu_{*}-2 \min _{\left[p_{0}, 0\right]} B\right)^{1 / 2}}<0 .
$$

Since $H(p ; \mu) \in[0, d]$ for $p \in\left[p_{0}, p_{\mu}\right]$ and $\bar{\rho}^{\prime} \leq 0$, it follows that

$$
\begin{aligned}
H\left(p_{\mu} ; \mu\right) & =\int_{p_{0}}^{p_{\mu}}\left(\mu-2 \int_{p_{0}}^{r}\left[g \bar{\rho}^{\prime}(s)(H(s)-d)+\beta(s)\right] \mathrm{d} s\right)^{-1 / 2} \mathrm{~d} r \\
& \geq \int_{p_{0}}^{p_{\mu}}\left(\mu-2 \int_{p_{0}}^{r} \beta(s) \mathrm{d} s\right)^{-1 / 2} \mathrm{~d} r \geq \frac{p_{\mu}-p_{0}}{\left(\mu-2 \min _{\left[p_{0}, 0\right]} B\right)^{1 / 2}} .
\end{aligned}
$$

In view of (3.10) we conclude (by a contradiction argument) that $p_{\mu}<0$ for all $\mu$ which are sufficiently close to $\mu_{*}$. Hence

$$
H(0 ; \mu)>d \quad \text { for } \mu \text { sufficiently close to } \mu_{*} .
$$

Together with Lemma 3.3 we conclude the following result establishing the existence of at least one strong laminar flow solution to (3.3) and (2.11).

Proposition 3.5 Assume that (3.10) is satisfied. Then there is at least one solution $H \in W_{r}^{2}\left(\left(p_{0}, 0\right)\right)$ to (3.4). Moreover, $H^{\prime}$ is a positive function.

Proof The proof follows from Proposition 3.2, Lemma 3.3, and the discussion preceding Proposition 3.5.

\section{Local bifurcation}

In the following we assume that the density and the Bernoulli function satisfy (2.1), (2.5), and (3.1), i.e.,

$$
\bar{\rho} \geq \rho_{0}, \bar{\rho}^{\prime} \leq 0 \quad \text { in }\left[p_{0}, 0\right], \quad \bar{\rho} \in W_{r}^{1}\left(\left(p_{0}, 0\right)\right), \beta \in L_{r}\left(\left(p_{0}, 0\right)\right), \quad r \in(1, \infty),
$$

and that (3.10) holds true. This guarantees, in particular, the existence of a laminar flow solution to (3.3) and (2.11).

The first goal of this section is to recast the weak formulation of (3.3) and (2.11) as an abstract bifurcation problem. For this goal only the Hölder regularity of the strong solutions to (3.3) and (2.11) is needed. To proceed, we define the Banach space 


$$
\mathbb{Y}_{1}:=\left\{\varphi+\partial_{q} \psi_{1}+\partial_{p} \psi_{2} \in \mathcal{D}^{\prime}(\Omega): \begin{array}{l}
\varphi \in L_{\infty}(\Omega), \psi_{1}, \psi_{2} \in \mathrm{C}^{\alpha}(\bar{\Omega}), \\
\varphi, \psi_{2} \text { are even, } \psi_{1} \text { is odd in } q
\end{array}\right\}
$$

which is endowed with the norm

$$
\|u\|_{\mathbb{\mho}_{1}}:=\inf \left\{\|\varphi\|_{\infty}+\left\|\psi_{1}\right\|_{\alpha}+\left\|\psi_{2}\right\|_{\alpha}: \begin{array}{l}
u=\varphi+\partial_{q} \psi_{1}+\partial_{p} \psi_{2}, \\
\varphi \in L_{\infty}(\Omega), \psi_{1}, \psi_{2} \in \mathrm{C}^{\alpha}(\bar{\Omega})
\end{array}\right\}
$$

and we set

$$
\begin{aligned}
& \mathbb{Y}_{2}:=\left\{\phi \in \mathrm{C}^{1+\alpha}(\mathbb{R}): \phi \text { is even }\right\}, \\
& \mathbb{X}:=\left\{h \in \mathrm{C}^{1+\alpha}(\bar{\Omega}): h \text { is even in } q \text { and } h=0 \text { on } p=p_{0}\right\} .
\end{aligned}
$$

We recall that these Banach spaces consist only of periodic distributions of period 1. Furthermore, we fix a laminar flow solution $H \in W_{r}^{2}\left(\left(p_{0}, 0\right)\right) \hookrightarrow \mathbb{X}$ (as found in Proposition 3.5) and we let $\mathcal{O}$ denote the open subset of $\mathbb{X}$ defined by

$$
\mathcal{O}:=\left\{h \in \mathbb{X}: \min _{\bar{\Omega}}\left(h_{p}+H^{\prime}\right)>0\right\} .
$$

The weak formulation of (3.3) and (2.11) can then be recast as the nonlinear and nonlocal equation

$$
\mathcal{F}(\lambda, h)=0,
$$

where $\mathcal{F}:=\left(\mathcal{F}_{1}, \mathcal{F}_{2}\right):(0, \infty) \times \mathcal{O} \rightarrow \mathbb{Y}:=\mathbb{Y}_{1} \times \mathbb{V}_{2}$ is given by

$$
\begin{gathered}
\mathcal{F}_{1}(\lambda, h):=\left(\frac{h_{q}}{h_{p}+H^{\prime}}\right)_{q}-\left(\frac{\lambda^{2}+h_{q}^{2}}{2\left(h_{p}+H^{\prime}\right)^{2}}+\lambda^{2} B+\lambda^{2} g \bar{\rho}(h+H-d)\right)_{p}+\lambda^{2} g \bar{\rho}\left(h_{p}+H^{\prime}\right), \\
\mathcal{F}_{2}(\lambda, h):=\operatorname{tr}_{0} h-\left(1-\partial_{q}^{2}\right)^{-1} \operatorname{tr}_{0}\left[h-\frac{\left(\lambda^{2}+h_{q}^{2}\right)^{3 / 2}}{2 \sigma \lambda^{3}}\left(\frac{\lambda^{2}+h_{q}^{2}}{\left(h_{p}+H^{\prime}\right)^{2}}+2 \lambda^{2} g \bar{\rho} h\right.\right. \\
\left.\left.-\int_{0}^{1} \frac{\lambda^{2}+h_{q}^{2}}{\left(h_{p}+H^{\prime}\right)^{2}} \mathrm{~d} q\right)\right] .
\end{gathered}
$$

We regard Eq. (4.1) as a bifurcation problem with bifurcation parameter $\lambda$. Recalling that $H$ solves (3.4), it holds that

$$
\mathcal{F}(\lambda, 0)=0 \quad \text { for all } \lambda>0 .
$$

We note that $\mathcal{F}$ is smooth with respect to its variables, that is

$$
\mathcal{F} \in \mathrm{C}^{\infty}((0, \infty) \times \mathcal{O}, \mathbb{Y})
$$

The aim is to apply the Crandall-Rabinowitz theorem [20, Theorem 1.7] on bifurcation from simple eigenvalues to (4.1) to determine other solutions to (4.1) that depend on the variable $q$. To this end we need to determine $\lambda_{*}>0$ such that the partial Fréchet derivative $\partial_{h} \mathcal{F}\left(\lambda_{*}, 0\right)$ is a Fredholm operator of index zero with a one-dimensional kernel. A certain transversality condition also needs to be satisfied, cf. Proposition 4.13. 
To begin, we observe that, given $\lambda>0$, the Fréchet derivative $\partial_{h} \mathcal{F}(\lambda, 0)=(L, T) \in \mathcal{L}(\mathbb{X}, \mathbb{Y})$ is expressed by

$$
\begin{aligned}
& L[h]=\left(\frac{h_{q}}{H^{\prime}}\right)_{q}+\lambda^{2}\left(\frac{h_{p}}{H^{\prime 3}}-g \bar{\rho} h\right)_{p}+\lambda^{2} g \bar{\rho} h_{p} \in \mathbb{Y}_{1}, \\
& T[h]=\operatorname{tr}_{0} h-\left(1-\partial_{q}^{2}\right)^{-1} \operatorname{tr}_{0}\left[h+\frac{\lambda^{2}}{\sigma}\left(\frac{h_{p}}{H^{\prime 3}}-g \bar{\rho} h-\int_{0}^{1} \frac{h_{p}}{H^{\prime 3}} \mathrm{~d} q\right)\right] \in \mathbb{Y}_{2}, \quad h \in \mathbb{X} .
\end{aligned}
$$

Lemma 4.1 Given $\lambda>0$, it holds that $\partial_{h} \mathcal{F}(\lambda, 0)$ is a Fredholm operator of index zero.

Proof According to [28, Theorem 8.34], the mapping

$$
\left[h \mapsto\left(\left(\frac{h_{q}}{H^{\prime}}\right)_{q}+\lambda^{2}\left(\frac{h_{p}}{H^{\prime 3}}\right)_{p}, \operatorname{tr}_{0} h\right)\right]: \mathbb{X} \rightarrow \mathbb{Y}
$$

is an isomorphism (we recall that $H^{\prime}$ is positive). Since $\left(1-\partial_{q}^{2}\right)^{-1} \in \mathcal{L}\left(\mathrm{C}(\mathbb{R}), \mathrm{C}^{2}(\mathbb{R})\right)$, it follows that the operator

$$
\left[h \mapsto\left(-\lambda^{2} g(\bar{\rho} h)_{p}+\lambda^{2} g \bar{\rho} h_{p},-\left(1-\partial_{q}^{2}\right)^{-1} \operatorname{tr}_{0}\left[h+\frac{\lambda^{2}}{\sigma}\left(\frac{h_{p}}{H^{\prime 3}}-g \bar{\rho} h-\int_{0}^{1} \frac{h_{p}}{H^{\prime 3}} \mathrm{~d} q\right)\right]\right)\right]
$$

is compact. Therefore, the desired claim follows.

We want to determine special values $\lambda_{*}$ of the wavelength parameter such that additionally the kernel of $\partial_{h} \mathcal{F}\left(\lambda_{*}, 0\right)$ is one-dimensional. Let thus $w \in \mathbb{X}$ be such that $\partial_{h} \mathcal{F}(\lambda, 0)[w]=0$. From $L[w]=0$ we find that the Fourier coefficient

$$
w_{k}(p):=\int_{0}^{1} w(q, p) \cos (2 k \pi q) \mathrm{d} q, \quad k \in \mathbb{N}, p \in\left[p_{0}, 0\right],
$$

satisfies the equation

$$
\lambda^{2}\left(\frac{1}{H^{\prime 3}} w_{k}^{\prime}-g \bar{\rho} w_{k}\right)^{\prime}+\lambda^{2} g \bar{\rho} w_{k}^{\prime}-\frac{(2 k \pi)^{2}}{H^{\prime}} w_{k}=0 \quad \text { in } L_{r}\left(\left(p_{0}, 0\right)\right) .
$$

Furthermore, since $T[w]=0$, we find that

$$
\left(\lambda^{2} g \bar{\rho}(0)+\sigma(2 k \pi)^{2}\right) w_{k}(0)=\frac{\lambda^{2}}{H^{\prime 3}(0)} w_{k}^{\prime}(0), \quad k \geq 1,
$$

and

$$
w_{0}(0)=0,
$$

respectively. Finally, since $w \in \mathbb{X}$, it holds that

$$
w_{k}\left(p_{0}\right)=0, \quad k \in \mathbb{N} .
$$

Hence, for $k=0$, we find that $w_{0} \in W_{r}^{2}\left(\left(p_{0}, 0\right)\right)$ solves the system 


$$
\left\{\begin{array}{l}
\left(a^{3} w_{0}^{\prime}\right)^{\prime}-g \bar{\rho}^{\prime} w_{0}=0 \quad \text { in } L_{r}\left(\left(p_{0}, 0\right)\right) \\
w_{0}\left(p_{0}\right)=w_{0}(0)=0
\end{array}\right.
$$

where

$$
a:=1 / H^{\prime} \in W_{r}^{1}\left(\left(p_{0}, 0\right)\right)
$$

Furthermore, given $k \geq 1$, the function $w_{k} \in W_{r}^{2}\left(\left(p_{0}, 0\right)\right)$ solves the system

$$
\left\{\begin{array}{l}
\lambda^{2}\left(a^{3} w^{\prime}\right)^{\prime}-\lambda^{2} g \bar{\rho}^{\prime} w-\vartheta a w=0 \quad \text { in } L_{r}\left(\left(p_{0}, 0\right)\right) \\
\left(\lambda^{2} g \bar{\rho}(0)+\sigma \vartheta\right) w(0)=\lambda^{2} a^{3}(0) w^{\prime}(0) \\
w\left(p_{0}\right)=0
\end{array}\right.
$$

with $\vartheta:=(2 k \pi)^{2}$.

The remainder of this section is organized as follows. In the first paragraph we specify a condition under which (4.4) has only the trivial solution $w_{0}=0$. The objective of the second paragraph is twofold. On the one hand we determine special values $\lambda_{*}$ of $\lambda$ such that (4.5) has a one-dimensional space of solutions for $k=1$, respectively $\vartheta=(2 \pi)^{2}$. On the other hand we prove that (4.5) admits only the trivial solution $w_{k}=0$ for $\vartheta \in\left\{(2 k \pi)^{2}: k \geq 2\right\}$. The third paragraph treats the above mentioned transversality condition and the last paragraph is devoted to the proof of Theorem 2.3.

Conditions such that $w_{0}=0$. We now show that, under some additional restrictions (cf. (4.7)), the system (4.4) has only the trivial zero solution. Equivalently formulated, we show that the elliptic operator $\left[u \mapsto\left(a^{3} u^{\prime}\right)^{\prime}-g \bar{\rho}^{\prime} u\right]$, which is supplemented by homogeneous Dirichlet boundary conditions, does not have zero as an eigenvalue. We point out that the coefficient of $u$ is positive and it may be unbounded, while the coefficient $a^{3}$ is not explicitly determined. This is where the assumption $(2.15)_{2}$ becomes important, as it constitutes an explicit relation in terms of $d, p_{0}, \beta$, and $\bar{\rho}$ which ensures that (4.7) is satisfied, see Example 4.3. We also emphasize that in the constant density case the assertion of Lemma 4.2 follows via maximum principles, hence (4.7) (or (4.12)) is then not needed. Moreover, the restriction (4.7) (or its weaker version (4.12)) is also used at several places in the paragraphs below in order to establish the existence of a bifurcation point. In particular, for homogeneous irrotational waves (that is $\bar{\rho}=\rho \in \mathbb{R}$ and $\beta=0$ ), see Remark 4.10, the explicit condition (4.12) ensures, due to the fact that $x_{*}<2$, that the dispersion relation (4.40) has at least a solution. Since $x_{*} \approx 1.9368$, this shows that (4.12) is close to being optimal with respect to this issue.

Lemma 4.2 Let

$$
A(p):=\int_{p_{0}}^{p} \frac{g \bar{\rho}}{a^{3}}(s) \mathrm{d} s, \quad p \in\left[p_{0}, 0\right],
$$

and assume that

$$
e^{2 A(0)}-2 A(0) \leq 5 .
$$

Then (4.4) has only the trivial solution $w_{0}=0$. 
Proof Let $w_{0}$ be a solution to (4.4). Then, letting $z_{0}:=a^{3} w_{0}^{\prime}-g \bar{\rho} w_{0}$ we may recast (4.4) as a linear system of first-order ODEs with continuous coefficients, namely

$$
\left\{\begin{array}{l}
w_{0}^{\prime}=A^{\prime} w_{0}+a^{-3} z_{0} \\
z_{0}^{\prime}=-g \bar{\rho} A^{\prime} w_{0}-A^{\prime} z_{0} .
\end{array}\right.
$$

To obtain a contradiction, we assume that $z_{0}\left(p_{0}\right)=a^{3}\left(p_{0}\right) w_{0}^{\prime}\left(p_{0}\right) \neq 0$ as the solution to (4.8) that satisfies $\left(w_{0}, z_{0}\right)\left(p_{0}\right)=(0,0)$ is the trivial one. Without loss of generality let $z_{0}\left(p_{0}\right)=: \alpha>0$. We next show that $z_{0}>0$ in $\left[p_{0}, 0\right]$ if (4.7) holds true. Since, by $(4.8)_{1}$,

$$
w_{0}(p) e^{-A(p)}=\int_{p_{0}}^{p} \frac{z_{0} e^{-A}}{a^{3}}(s) \mathrm{d} s, \quad p \in\left[p_{0}, 0\right],
$$

this then contradicts the boundary condition $w_{0}(0)=0$ and the proof is complete.

To prove that $z_{0}$ is positive in $\left[p_{0}, 0\right]$, we assume there exists $p_{1} \in\left(p_{0}, 0\right]$ with $z_{0}>0$ in $\left[p_{0}, p_{1}\right)$ and $z_{0}\left(p_{1}\right)=0$. The relation (4.9) implies that $w_{0}>0$ in $\left(p_{0}, p_{1}\right]$. Invoking $(4.8)_{2}$ it holds that

$$
z_{0}(p) e^{A(p)}=\alpha-\int_{p_{0}}^{p} g \bar{\rho}(s) A^{\prime}(s) e^{2 A(s)} \int_{p_{0}}^{s} \frac{z_{0}(r) e^{-A(r)}}{a^{3}(r)} \mathrm{d} r \mathrm{~d} s, \quad p \in\left[p_{0}, 0\right] .
$$

Since $A^{\prime}$ is positive, we find that $z_{0} e^{A}$ is decreasing in $\left[p_{0}, p_{1}\right]$. Consequently

$$
0 \leq z_{0}(p)<\alpha e^{-A(p)}, \quad p \in\left(p_{0}, p_{1}\right] .
$$

Since $z_{0}\left(p_{1}\right)=0$, the relation (4.10) yields

$$
\alpha=\int_{p_{0}}^{p_{1}} g \bar{\rho}(s) A^{\prime}(s) e^{2 A(s)} \int_{p_{0}}^{s} \frac{z_{0}(r) e^{-A(r)}}{a^{3}(r)} \mathrm{d} r \mathrm{~d} s,
$$

and using (4.11) we arrive at

$$
1<\int_{p_{0}}^{p_{1}} g \bar{\rho}(s) A^{\prime}(s) e^{2 A(s)} \int_{p_{0}}^{s} \frac{e^{-2 A(r)}}{a^{3}(r)} \mathrm{d} r \mathrm{~d} s .
$$

Since $\bar{\rho}(s) \leq \bar{\rho}(r)$ for $p_{0} \leq r \leq s \leq 0$ and recalling the definition of $A$ we get in view of (4.7) that

$$
\begin{aligned}
1 & <\int_{p_{0}}^{p_{1}} A^{\prime}(s) e^{2 A(s)} \int_{p_{0}}^{s} A^{\prime}(r) e^{-2 A(r)} \mathrm{d} r \mathrm{~d} s=\frac{1}{2} \int_{p_{0}}^{p_{1}} A^{\prime}(s)\left(e^{2 A(s)}-1\right) \mathrm{d} s \\
& \leq \frac{1}{4}\left(e^{2 A(0)}-2 A(0)-1\right) \leq 1,
\end{aligned}
$$

which is a contradiction. Our assumption is thus false and the proof complete.

We now provide a quantitative condition which ensures that (4.7) is satisfied.

Example 4.3 Let $\mu_{*}$ be as defined in (2.15) and assume that (3.10) holds. If 


$$
\frac{g d^{3} \bar{\rho}\left(p_{0}\right)\left|p_{0}\right|}{\left[p_{0}^{2}-\left(\mu_{*}-\underset{\left[p_{0}, 0\right]}{2 \min B}\right) d^{2}\right]^{3 / 2}} \leq \frac{x_{*}}{2}
$$

where $x_{*} \approx 1.9368$ is the positive solution to

$$
e^{x}-x=5,
$$

then (4.7) is satisfied.

Proof Recall that $a=1 / H^{\prime}>0$ in $\left[p_{0}, 0\right]$ with $H$ being the solution to (3.4) that we fixed earlier. Since $H\left(p_{0}\right)=0$ and $H(0)=d$, there exists $p_{1} \in\left[p_{0}, 0\right]$ such that $H^{\prime}\left(p_{1}\right) \leq d /\left|p_{0}\right|$. Integration of (3.4) $)_{1}$ over $\left[p_{1}, p\right]$, with $p \in\left[p_{0}, 0\right]$ arbitrary, yields

$$
\begin{aligned}
a^{2}(p) & =a^{2}\left(p_{1}\right)-2 \int_{p_{1}}^{p}\left[g \bar{\rho}^{\prime}(s)(H(s)-d)+\beta(s)\right] \mathrm{d} s \\
& \geq \frac{p_{0}^{2}}{d^{2}}-2 \int_{p_{0}}^{0} g d\left|\rho^{\prime}(s)\right| \mathrm{d} s-2 B(p)+2 B\left(p_{1}\right) \\
& \geq \frac{p_{0}^{2}}{d^{2}}-\left(\mu_{*}-2 \min _{\left[p_{0}, 0\right]} B\right),
\end{aligned}
$$

the positivity of the constant on the right-hand side of this inequality being equivalent to (3.10). Consequently,

$$
\frac{1}{a(p)} \leq \frac{d}{\sqrt{p_{0}^{2}-\left(\mu_{*}-2 \min _{\left[p_{0}, 0\right]} B\right) d^{2}}}, \quad p \in\left[p_{0}, 0\right],
$$

and, recalling that $\bar{\rho} \leq \bar{\rho}\left(p_{0}\right)$, we get

$$
A(0)=\int_{p_{0}}^{0} \frac{g \bar{\rho}(s)}{a^{3}(s)} \mathrm{d} s \leq g \bar{\rho}\left(p_{0}\right) \int_{p_{0}}^{0} \frac{1}{a^{3}(s)} \mathrm{d} s \leq \frac{g d^{3} \bar{\rho}\left(p_{0}\right)\left|p_{0}\right|}{\left[p_{0}^{2}-\left(\mu_{*}-\underset{\left[p_{0}, 0\right]}{2 \min B}\right) d^{2}\right]^{3 / 2}} \leq \frac{x_{*}}{2} .
$$

Hence (4.7) holds true.

Relation (4.12) provides an explicit condition which ensures that the system (4.4) has only the trivial solution $w_{0}=0$. Consequently, for all $\lambda>0$, the kernel of $\partial_{h} \mathcal{F}(\lambda, 0)$ does not contain functions that depend only on the variable $p$ (except for the zero function). We now address the second issue of determining $\lambda_{*}>0$ such that $\partial_{h} \mathcal{F}\left(\lambda_{*}, 0\right)$ has a onedimensional kernel spanned by a function of the form $w_{1}(p) \cos (2 \pi q)$, with $w_{1}$ being (up to a multiplicative constant) the only nontrivial solution to (4.5) when $\lambda=\lambda_{*}$ and $\vartheta \in\left\{(2 k \pi)^{2}: k \in \mathbb{N}\right\}$.

The system (4.5) with $\vartheta$ as a variable. We seek $\lambda>0$ such that (4.5) has a one-dimensional space of solutions for $\vartheta=(2 \pi)^{2}$ and only the trivial solution for $\vartheta>(2 \pi)^{2}$. To this end we first determine the dimension of the space of solutions to (4.5). Given $\lambda \in(0, \infty)$ 
and $\vartheta \in \mathbb{R}$, we let $R_{\lambda, \vartheta}: W_{r, 0}^{2}\left(\left(p_{0}, 0\right)\right) \rightarrow L_{r}\left(\left(p_{0}, 0\right)\right) \times \mathbb{R}$ denote the Sturm-Liouville-type operator

$$
R_{\lambda, \vartheta}[w]:=\left(\begin{array}{l}
\lambda^{2}\left(a^{3} w^{\prime}\right)^{\prime}-\lambda^{2} g \bar{\rho}^{\prime} w-\vartheta a w \\
\lambda^{2} a^{3}(0) w^{\prime}(0)-\left(\lambda^{2} g \bar{\rho}(0)+\sigma \vartheta\right) w(0)
\end{array}\right)
$$

where we set

$$
W_{r, 0}^{2}\left(\left(p_{0}, 0\right)\right)=\left\{w \in W_{r}^{2}\left(\left(p_{0}, 0\right)\right): w\left(p_{0}\right)=0\right\} .
$$

We associate with (4.5) the initial value problems

$$
\left\{\begin{array}{l}
\lambda^{2}\left(a^{3} \widetilde{w}_{1}^{\prime}-g \bar{\rho} \widetilde{w}_{1}\right)^{\prime}+\lambda^{2} g \bar{\rho} \widetilde{w}_{1}^{\prime}-\vartheta a \widetilde{w}_{1}=0 \quad \text { in } L_{r}\left(\left(p_{0}, 0\right)\right), \\
\widetilde{w}_{1}\left(p_{0}\right)=0, \quad \widetilde{w}_{1}^{\prime}\left(p_{0}\right)=1,
\end{array}\right.
$$

and

$$
\left\{\begin{array}{l}
\lambda^{2}\left(a^{3} \widetilde{w}_{2}^{\prime}-g \bar{\rho} \widetilde{w}_{2}\right)^{\prime}+\lambda^{2} g \bar{\rho} \widetilde{w}_{2}^{\prime}-\vartheta a \widetilde{w}_{2}=0 \quad \text { in } L_{r}\left(\left(p_{0}, 0\right)\right), \\
\widetilde{w}_{2}(0)=\lambda^{2} a^{3}(0), \quad \widetilde{w}_{2}^{\prime}(0)=\lambda^{2} g \bar{\rho}(0)+\sigma \vartheta
\end{array}\right.
$$

Lemma 4.4 Given $\lambda>0$ and $\vartheta \in \mathbb{R}$, the operator $R_{\lambda, \vartheta}: W_{r, 0}^{2}\left(\left(p_{0}, 0\right)\right) \rightarrow L_{r}\left(\left(p_{0}, 0\right)\right) \times \mathbb{R}$ defined in (4.15) is a Fredholm operator of index zero and $\operatorname{dim} \operatorname{ker} R_{\lambda, \vartheta} \leq 1$. Furthermore, $\operatorname{dim} \operatorname{ker} R_{\lambda, 9}=1$ if and only if the solutions $\widetilde{w}_{1}$ and $\widetilde{w}_{2}$ to (4.16) and (4.17) are linearly dependent. In this case it holds that

$$
\operatorname{ker} R_{\lambda, 9}=\operatorname{span}\left\{\tilde{w}_{1}\right\}=\operatorname{span}\left\{\tilde{w}_{2}\right\} .
$$

Proof We first decompose $R_{\lambda, \vartheta}=R_{I}+R_{c}$, where

$$
R_{I}[w]:=\left(\begin{array}{l}
\lambda^{2}\left(a^{3} w^{\prime}\right)^{\prime} \\
\lambda^{2} a^{3}(0) w^{\prime}(0)
\end{array}\right) \quad \text { and } \quad R_{c}[w]:=\left(\begin{array}{l}
-\vartheta a w-\lambda^{2} g \bar{\rho}^{\prime} w \\
-\left(\lambda^{2} g \bar{\rho}(0)+\sigma \vartheta\right) w(0)
\end{array}\right) .
$$

It is clear that $R_{c}$ is a compact operator. Furthermore, $R_{I}$ is an isomorphism. Hence, $R_{\lambda, \vartheta}$ is a Fredholm operator of index zero.

We now set $z_{i}:=a^{3} \widetilde{w}_{i}^{\prime}-g \bar{\rho} \widetilde{w}_{i}, i \in\{1,2\}$, where $\widetilde{w}_{1}$ and $\widetilde{w}_{2}$ denote the unknowns in (4.16) and (4.17), respectively. Recalling the definition of $A$ in (4.6), Eqs. (4.16) 1 and $(4.17)_{1}$ can be recast as a first-order system of linear ODEs with continuous coefficients:

$$
\left\{\begin{array}{l}
\widetilde{w}_{i}^{\prime}:=A^{\prime} \widetilde{w}_{i}+a^{-3} z_{i}, \\
z_{i}^{\prime}=\left(\lambda^{-2} \vartheta a-g \bar{\rho} A^{\prime}\right) \widetilde{w}_{i}-A^{\prime} z_{i}
\end{array} \quad \text { for } i \in\{1,2\},\right.
$$

and the classical theory, cf., e.g., [2, Proposition 7.8], ensures that each of the problems (4.16) and (4.17) has a unique solution $\widetilde{w}_{i} \in W_{r}^{2}\left(\left(p_{0}, 0\right)\right), i \in\{1,2\}$. Moreover, given $w_{a}, w_{b} \in W_{r}^{2}\left(\left(p_{0}, 0\right)\right)$ solutions to $(4.5)_{1}$, it follows that

$$
a^{3}\left(w_{a}^{\prime} w_{b}-w_{a} w_{b}^{\prime}\right)=C \quad \text { in }\left[p_{0}, 0\right]
$$

for some $C \in \mathbb{R}$. Hence $w_{a}$ and $w_{b}$ are colinear if they also belong to $W_{r, 0}^{2}\left(\left(p_{0}, 0\right)\right)$. This proves in particular that $\operatorname{dim} \operatorname{ker} R_{\lambda, \vartheta} \leq 1$. It remains to establishing the last claim. Let $\operatorname{dim} \operatorname{ker} R_{\lambda, \vartheta}=1$ and choose $0 \neq w \in \operatorname{ker} R_{\lambda, \vartheta}$. Relation (4.19) implies that $w$ and $\widetilde{w}_{1}$ are 
colinear. Invoking $(4.15)_{2}$ and $(4.17)_{2},(4.19)$ shows that also $w$ and $\widetilde{w}_{2}$ are colinear. Finally, if $\widetilde{w}_{1}$ and $\widetilde{w}_{2}$ are colinear, it is easy to see that they both belong to $\operatorname{ker} R_{\lambda, \vartheta}$.

We can now reformulate our task as the problem of determining $\lambda_{*}>0$ such that the Wronskian

$$
W(\cdot ; \lambda, \vartheta):=\left|\begin{array}{ll}
\widetilde{w}_{1} & \widetilde{w}_{2} \\
\widetilde{w}_{1}^{\prime} & \widetilde{w}_{2}^{\prime}
\end{array}\right|
$$

vanishes in $\left[p_{0}, 0\right]$ only for $\vartheta=(2 \pi)^{2}$. Recalling (4.19), it follows that $W(\cdot ; \lambda, \vartheta)$ vanishes in $\left[p_{0}, 0\right]$ if and only if it vanishes at $p=0$. For this reason we consider the function $W(0 ; \cdot, \cdot):(0, \infty) \times \mathbb{R} \rightarrow \mathbb{R}$ defined by

$$
\begin{aligned}
W(0 ; \lambda, \vartheta) & =\widetilde{w}_{1}(0) \widetilde{w}_{2}^{\prime}(0)-\widetilde{w}_{1}^{\prime}(0) \widetilde{w}_{2}(0)=\left(\lambda^{2} g \bar{\rho}(0)+\sigma \vartheta\right) \widetilde{w}_{1}(0)-\lambda^{2} a^{3}(0) \widetilde{w}_{1}^{\prime}(0) \\
& =\sigma \vartheta \widetilde{w}_{1}(0)-\lambda^{2} z_{1}(0),
\end{aligned}
$$

where $z_{1}=a^{3} \widetilde{w}_{1}^{\prime}-g \bar{\rho} \widetilde{w}_{1}$ is the new variable introduced in (4.18). By [2, Proposition 9.5] it holds that

$$
W(0 ; \cdot, \cdot) \in \mathrm{C}^{\infty}((0, \infty) \times \mathbb{R}) .
$$

We next prove that for each $\lambda>0$ there exists at least one solution $\vartheta$ to $W(0 ; \lambda, \vartheta)=0$. As a first step we show that

$$
W(0 ; \lambda, 0)=-\lambda^{2} z_{1}(0)<0 .
$$

This is a direct consequence of the following more general statement.

Lemma 4.5 Assume that (4.7) is satisfied. If $\vartheta=0$ and $\lambda>0$, then $z_{1}>0, \widetilde{w}_{1}^{\prime}>0$ in $\left[p_{0}, 0\right]$ and $\widetilde{w}_{1}>0$ in $\left(p_{0}, 0\right]$.

Proof Arguing as in the proof of Lemma 4.2 it follows that $z_{1}>0$ in $\left[p_{0}, 0\right]$. The remaining claims are direct consequences of the latter property. Indeed, the relations

$$
\widetilde{w}_{1}^{\prime}-A^{\prime} \widetilde{w}_{1}=\frac{z_{1}}{a^{3}} \quad \text { in }\left[p_{0}, 0\right], \quad \widetilde{w}_{1}\left(p_{0}\right)=0,
$$

imply

$$
\widetilde{w}_{1}(p)=\int_{p_{0}}^{p} \frac{z_{1}(s)}{a^{3}(s)} e^{A(p)-A(s)} \mathrm{d} s, \quad p \in\left[p_{0}, 0\right] .
$$

As $z_{1}$ is positive, we conclude that $\widetilde{w}_{1}>0$ in $\left(p_{0}, 0\right]$ and $\widetilde{w}_{1}^{\prime}>0$ in $\left[p_{0}, 0\right]$.

In view of (4.21), for the existence of a solution $\vartheta$ to $W(0 ; \lambda, \vartheta)=0$ it thus suffices to prove that $W(0 ; \lambda, \vartheta) \rightarrow \infty$ for $\vartheta \rightarrow \infty$. We first show that if $\vartheta / \lambda^{2}$ is sufficiently large, then $\widetilde{w}_{1}^{\prime}$ is a positive function. This property is not obvious because of the fact that $\bar{\rho}^{\prime}$ has not only the opposed sign in (4.5) but it can also be unbounded. However, when considering the equivalent formulation (4.18), this feature follows quite naturally.

Lemma 4.6 Let $\lambda>0$ and assume that 


$$
\frac{\vartheta}{\lambda^{2}} \geq \frac{g^{2} \bar{\rho}^{2}\left(p_{0}\right)}{\min _{\left[p_{0}, 0\right]} a^{4}}
$$

Then $z_{1} e^{A}$ and $\widetilde{w}_{1}$ are increasing functions and it holds that

$$
z_{1}(p) \geq a^{3}\left(p_{0}\right) e^{-A(p)}>0, \quad \text { for all } p \in\left[p_{0}, 0\right] .
$$

Proof Integrating (4.18) (with $i=1$ ) yields

$$
\widetilde{w}_{1}(p)=\int_{p_{0}}^{p} \frac{z_{1}(s)}{a^{3}(s)} e^{A(p)-A(s)} \mathrm{d} s
$$

and

$$
z_{1}(p)=a^{3}\left(p_{0}\right) e^{-A(p)}+\int_{p_{0}}^{p}\left(\frac{\vartheta a(s)}{\lambda^{2}}-g \bar{\rho}(s) A^{\prime}(s)\right) e^{A(s)-A(p)} \int_{p_{0}}^{s} \frac{z_{1}(r)}{a^{3}(r)} e^{A(s)-A(r)} \mathrm{d} r \mathrm{~d} s
$$

for all $p \in\left[p_{0}, 0\right]$. Hence, the assertions follow due to (4.24).

Combining (4.20) and (4.26) yields

$$
\begin{aligned}
W(0 ; \lambda, \vartheta)= & \sigma \vartheta \int_{p_{0}}^{0} \frac{z_{1}(s)}{a^{3}(s)} e^{A(0)-A(s)} \mathrm{d} s \\
& -\lambda^{2}\left[a^{3}\left(p_{0}\right) e^{-A(0)}+\int_{p_{0}}^{0}\left(\frac{\vartheta a(s)}{\lambda^{2}}-g \bar{\rho}(s) A^{\prime}(s)\right) \int_{p_{0}}^{s} \frac{z_{1}(r)}{a^{3}(r)} \frac{e^{2 A(s)}}{e^{A(0)+A(r)}} \mathrm{d} r \mathrm{~d} s\right] .
\end{aligned}
$$

Lemma 4.7 Let $\lambda>0$ be given and assume that condition (4.7) is satisfied. It then holds:

$$
W(0 ; \lambda, \vartheta) \rightarrow \infty \quad \text { as } \quad \vartheta \rightarrow \infty .
$$

Proof In the following, we assume that $\vartheta$ is large enough to ensure (4.24). Recall that, by Lemma 4.6, the function $z_{1}$ is positive and $z_{1} e^{A}$ is increasing on $\left[p_{0}, 0\right]$. Using (4.27), we obtain the estimate

$$
W(0 ; \lambda, \vartheta) \geq C_{1} \vartheta\left(\int_{p_{0}}^{0} z_{1}(s) \mathrm{d} s+C_{2} \int_{p_{0}}^{0} s z_{1}(s) \mathrm{d} s\right)-\lambda^{2} a^{3}\left(p_{0}\right) e^{-A(0)}
$$

with positive constants $C_{1}$ and $C_{2}$ independent of $\vartheta$. Therefore it suffices to show that

$$
\liminf _{\vartheta \rightarrow \infty} \int_{p_{0}}^{0}\left(1+C_{2} s\right) z_{1}(s) \mathrm{d} s>0 .
$$

Let $p_{1} \in\left[p_{0}, 0\right]$ be an arbitrary number that is specified later. For $\vartheta$ sufficiently large it holds that

$$
\left(z_{1}(p) e^{A(p)}\right)^{\prime}=\left(\frac{\vartheta a(p)}{\lambda^{2}}-g \bar{\rho}(p) A^{\prime}(p)\right) \int_{p_{0}}^{p} \frac{z_{1}(s) e^{A(s)}}{a^{3}(s)} e^{2(A(p)-A(s))} \mathrm{d} s \geq C_{3} \frac{\vartheta}{\lambda^{2}} \int_{p_{1}}^{p} z_{1}(s) e^{A(s)} \mathrm{d} s
$$

for all $p \in\left[p_{1}, 0\right]$ with a positive constant $C_{3}$ independent of $\vartheta$. We define 


$$
Z(p):=\int_{p_{1}}^{p} z_{1}(s) e^{A(s)} \mathrm{d} s \quad \text { for all } p \in\left[p_{1}, 0\right]
$$

and observe that

$$
Z^{\prime \prime} \geq C_{3} \frac{\vartheta}{\lambda^{2}} Z \quad \text { on }\left[p_{1}, 0\right], \quad Z\left(p_{1}\right)=0, \quad Z^{\prime}\left(p_{1}\right)=z_{1}\left(p_{1}\right) e^{A\left(p_{1}\right)} .
$$

Now, let $U$ denote the solution of the initial value problem

$$
U^{\prime \prime}=C_{3} \frac{\vartheta}{\lambda^{2}} U \quad \text { on }\left[p_{1}, 0\right], \quad U\left(p_{1}\right)=0, \quad U^{\prime}\left(p_{1}\right)=z_{1}\left(p_{1}\right) e^{A\left(p_{1}\right)} .
$$

Then, $U$ is explicitly given by

$$
U(p)=\frac{z_{1}\left(p_{1}\right) e^{A\left(p_{1}\right)}}{\mu} \sinh \left(\mu\left(p-p_{1}\right)\right) \quad \text { with } \quad \mu:=\sqrt{C_{3} \frac{\vartheta}{\lambda^{2}}}
$$

for all $p \in\left[p_{1}, 0\right]$. It is straightforward to check that $Z \geq U$ on $\left[p_{1}, 0\right]$. As $z_{1}$ is positive and $A$ is increasing, we can conclude that

$$
\int_{p_{1}}^{0} z_{1}(s) \mathrm{d} s \geq e^{-A(0)} Z(0) \geq e^{-A(0)} U(0) .
$$

Now, we fix

$$
p_{1}:=\max \left\{-\frac{1}{2 C_{2}}, \frac{p_{0}}{2}\right\} .
$$

Then, since $z_{1}$ is positive and $\left(z_{1} e^{A}\right)$ is increasing, we use (4.31) and the definition of $p_{1}$ to derive the estimate

$$
\begin{aligned}
\int_{p_{0}}^{0}\left(1+C_{2} s\right) z_{1}(s) \mathrm{d} s & =\int_{p_{0}}^{p_{1}}\left(1+C_{2} s\right) z_{1}(s) \mathrm{d} s+\int_{p_{1}}^{0}\left(1+C_{2} s\right) z_{1}(s) \mathrm{d} s \\
& \geq \frac{1}{2} \int_{p_{1}}^{0} z_{1}(s) \mathrm{d} s-C_{4} \int_{p_{0}}^{p_{1}} e^{A(s)} z_{1}(s) \mathrm{d} s \\
& \geq \frac{1}{2}\left(e^{-A(0)} U(0)-C_{4}\left|p_{0}\right| e^{A\left(p_{1}\right)} z_{1}\left(p_{1}\right)\right) \\
& =\frac{z_{1}\left(p_{1}\right)}{2}\left(\frac{e^{A\left(p_{1}\right)-A(0)}}{\mu} \sinh \left(\mu\left|p_{1}\right|\right)-C_{4}\left|p_{0}\right| e^{A\left(p_{1}\right)}\right),
\end{aligned}
$$

where $C_{4}$ denotes a nonnegative constant that is independent of $\vartheta$. Hence, recalling (4.25), we observe that the right-hand side tends to infinity as $\vartheta \rightarrow \infty$. In particular, the assertion (4.30) directly follows.

The relations (4.21), (4.22), and (4.28) ensure that the equation $W(0 ; \lambda, \vartheta)=0$ has at least one solution $\vartheta>0$ for any fixed $\lambda>0$. The next result provides a remarkable identity, cf. (4.32), that will enable us later to identify the largest solution $\vartheta(\lambda)$ to the above equation in a quite explicit way. 
Lemma 4.8 Assume that (3.10) and (4.12) hold and that $(\lambda, \vartheta) \in(0, \infty)^{2}$ satisfies $W(0 ; \lambda, \vartheta)=0$. Then, it holds $\widetilde{w}_{1}(0) W_{\lambda}(0 ; \lambda, \vartheta)<0$ and

$$
\frac{W_{\vartheta}(0 ; \lambda, \vartheta)}{W_{\lambda}(0 ; \lambda, \vartheta)}=-\frac{\lambda}{2 \vartheta}<0 \text {. }
$$

Proof Let $\widetilde{w}_{1}=\widetilde{w}_{1}(\cdot ; \lambda, \vartheta)$ denote the solution of (4.16) corresponding to $\lambda$ and $\vartheta$. We first consider the derivative $W_{\lambda}(0 ; \lambda, \vartheta)$. Using the algebra property of $W_{r}^{1}\left(\left(p_{0}, 0\right)\right)$ we conclude that the partial derivative $\widetilde{w}_{1, \lambda}=\partial_{\lambda} \widetilde{w}_{1}(\cdot, \lambda, \vartheta)$ belongs to $W_{r}^{2}\left(\left(p_{0}, 0\right)\right)$ and solves the problem

$$
\left\{\begin{array}{l}
\lambda^{2}\left(a^{3} \widetilde{w}_{1, \lambda}^{\prime}\right)^{\prime}-\lambda^{2} g \bar{\rho}^{\prime} \widetilde{w}_{1, \lambda}-\vartheta a \widetilde{w}_{1, \lambda}=-2 \lambda\left(a^{3} \widetilde{w}_{1}^{\prime}\right)^{\prime}+2 \lambda g \bar{\rho}^{\prime} \widetilde{w}_{1} \quad \text { in } L_{r}\left(\left(p_{0}, 0\right)\right), \\
\widetilde{w}_{1, \lambda}\left(p_{0}\right)=0, \quad \widetilde{w}_{1, \lambda}^{\prime}\left(p_{0}\right)=0 .
\end{array}\right.
$$

We multiply $\left(4.16_{1}\right.$ by $\left.\widetilde{w}_{1, \lambda}\right)$ and $(4.33)_{1}$ by $\widetilde{w}_{1}$ and subtract the resulting equations. This yields

$$
\lambda^{2}\left(a^{3} \widetilde{w}_{1}^{\prime}\right)^{\prime} \widetilde{w}_{1, \lambda}-\lambda^{2}\left(a^{3} \widetilde{w}_{1, \lambda}^{\prime}\right)^{\prime} \widetilde{w}_{1}=2 \lambda\left(a^{3} \widetilde{w}_{1}^{\prime}\right)^{\prime} \widetilde{w}_{1}-2 \lambda g \bar{\rho}^{\prime} \widetilde{w}_{1}^{2} \quad \text { in } L_{r}\left(\left(p_{0}, 0\right)\right) .
$$

Integrating with respect to $p$ from $p_{0}$ to 0 and using integration by parts then gives

$$
\begin{aligned}
& \lambda^{2} a^{3}(0) \widetilde{w}_{1}^{\prime}(0) \widetilde{w}_{1, \lambda}(0)-\lambda^{2} a^{3}(0) \widetilde{w}_{1, \lambda}^{\prime}(0) \widetilde{w}_{1}(0) \\
& \quad=2 \lambda a^{3}(0) \widetilde{w}_{1}^{\prime}(0) \widetilde{w}_{1}(0)-2 \lambda \int_{p_{0}}^{0}\left[a^{3}(p)\left(\widetilde{w}_{1}^{\prime}\right)^{2}(p)+g \bar{\rho}^{\prime}(p) \widetilde{w}_{1}^{2}(p)\right] \mathrm{d} p .
\end{aligned}
$$

Recall that $W(0 ; \lambda, \vartheta)=\left(\lambda^{2} g \bar{\rho}(0)+\sigma \vartheta\right) \widetilde{w}_{1}(0)-\lambda^{2} a^{3}(0) \widetilde{w}_{1}^{\prime}(0)$ according to (4.20). Hence, the derivative with respect to $\lambda$ is given by

$$
W_{\lambda}(0 ; \lambda, \vartheta)=2 \lambda g \bar{\rho}(0) \widetilde{w}_{1}(0)+\left(\lambda^{2} g \bar{\rho}(0)+\sigma \vartheta\right) \widetilde{w}_{1, \lambda}(0)-2 \lambda a^{3}(0) \widetilde{w}_{1}^{\prime}(0)-\lambda^{2} a^{3}(0) \widetilde{w}_{1, \lambda}^{\prime}(0) .
$$

We point out that, since $W(0 ; \lambda, \vartheta)=0$, the functions $\widetilde{w}_{1}$ and $\widetilde{w}_{2}$ are linearly dependent and not identically zero. This implies that $\widetilde{w}_{1}(0) \neq 0$. Multiplying the latter identity by $\widetilde{w}_{1}(0)$ and using (4.34) and the colinearity of $\widetilde{w}_{1}$ and $\widetilde{w}_{2}$ we then obtain

$$
\widetilde{w}_{1}(0) W_{\lambda}(0 ; \lambda, \vartheta)=2 \lambda\left(g \bar{\rho}(0) \widetilde{w}_{1}^{2}(0)-\int_{p_{0}}^{0} a^{3}(p)\left(\widetilde{w}_{1}^{\prime}(p)\right)^{2} \mathrm{~d} p-\int_{p_{0}}^{0} g \bar{\rho}^{\prime}(p) \widetilde{w}_{1}^{2}(p) \mathrm{d} p\right) .
$$

In view of

$$
-\int_{p_{0}}^{0} g \bar{\rho}^{\prime}(p) \widetilde{w}_{1}^{2}(p) \mathrm{d} p \leq g\left(\bar{\rho}\left(p_{0}\right)-\bar{\rho}(0)\right)\left\|\widetilde{w}_{1}\right\|_{\mathrm{C}\left(\left[p_{0}, 0\right]\right)}^{2}
$$

we conclude that 


$$
\begin{aligned}
& \widetilde{w}_{1}(0) W_{\lambda}(0 ; \lambda, \vartheta) \\
& \quad \leq 2 \lambda\left(g \bar{\rho}(0)\left[\widetilde{w}_{1}^{2}(0)-\left\|\widetilde{w}_{1}\right\|_{\mathrm{C}\left(\left[p_{0}, 0\right]\right)}^{2}\right]+g \bar{\rho}\left(p_{0}\right)\left\|\widetilde{w}_{1}\right\|_{\mathrm{C}\left(\left[p_{0}, 0\right]\right)}^{2}-\int_{p_{0}}^{0} a^{3}(p)\left(\widetilde{w}_{1}^{\prime}(p)\right)^{2} \mathrm{~d} p\right) \\
& \quad \leq 2 \lambda\left(g \bar{\rho}\left(p_{0}\right)\left\|\widetilde{w}_{1}\right\|_{\mathrm{C}\left(\left[p_{0}, 0\right]\right)}^{2}-\int_{p_{0}}^{0} a^{3}(p)\left(\widetilde{w}_{1}^{\prime}(p)\right)^{2} \mathrm{~d} p\right) .
\end{aligned}
$$

Finally, choosing $p_{1} \in\left(p_{0}, 0\right]$ such that $\left|\widetilde{w}_{1}\left(p_{1}\right)\right|=\left\|\widetilde{w}_{1}\right\|_{\mathrm{C}\left(\left[p_{0}, 0\right]\right)}$, together with (4.14) (recall that the positive solution $x_{*}$ to $(4.13)$ satisfies $\left.x_{*}<2\right)$ we get

$$
\begin{aligned}
g \bar{\rho}\left(p_{0}\right)\left\|\widetilde{w}_{1}\right\|_{\mathrm{C}\left(\left[p_{0}, 0\right]\right)}^{2} & =g \bar{\rho}\left(p_{0}\right)\left(\int_{p_{0}}^{p_{1}} \widetilde{w}_{1}^{\prime}(p) \mathrm{d} p\right)^{2} \\
& \leq g \bar{\rho}\left(p_{0}\right)\left(\int_{p_{0}}^{0} \frac{1}{a^{3}(p)} \mathrm{d} p\right)\left(\int_{p_{0}}^{0} a^{3}(p)\left(\widetilde{w}_{1}^{\prime}(p)\right)^{2} \mathrm{~d} p\right) \\
& <\int_{p_{0}}^{0} a^{3}(p)\left(\widetilde{w}_{1}^{\prime}(p)\right)^{2} \mathrm{~d} p,
\end{aligned}
$$

and this proves that $\widetilde{w}_{1}(0) W_{\lambda}(0 ; \lambda, \vartheta)<0$.

We next consider $W_{\vartheta}(0 ; \lambda, \vartheta)$. The partial derivative $\widetilde{w}_{1, \vartheta}=\partial_{\vartheta} \widetilde{w}_{1}(\cdot, \lambda, \vartheta)$ belongs to $W_{r}^{2}\left(\left(p_{0}, 0\right)\right)$ and solves the problem

$$
\left\{\begin{array}{l}
\lambda^{2}\left(a^{3} \widetilde{w}_{1, \vartheta}^{\prime}\right)^{\prime}-\lambda^{2} g \bar{\rho}^{\prime} \widetilde{w}_{1, \vartheta}-\vartheta a \widetilde{w}_{1, \vartheta}=a \widetilde{w}_{1} \quad \text { in } L_{r}\left(\left(p_{0}, 0\right)\right), \\
\widetilde{w}_{1, \vartheta}\left(p_{0}\right)=0, \quad \widetilde{w}_{1, \vartheta}^{\prime}\left(p_{0}\right)=0 .
\end{array}\right.
$$

Multiplying $\left(4.16_{1}\right.$ by $\left.\widetilde{w}_{1, \vartheta}\right)$ and $\left(4.36_{1}\right.$ by $\left.\widetilde{w}_{1}\right)$ and subtracting the resulting equations gives

$$
\lambda^{2}\left(a^{3} \widetilde{w}_{1}^{\prime}\right)^{\prime} \widetilde{w}_{1, \vartheta}-\lambda^{2}\left(a^{3} \widetilde{w}_{1, \vartheta}^{\prime}\right)^{\prime} \widetilde{w}_{1}=-a \widetilde{w}_{1}^{2} \quad \text { in } L_{r}\left(\left(p_{0}, 0\right)\right) .
$$

Integrating with respect to $p$ from $p_{0}$ to 0 and using integration by parts, we infer that

$$
\lambda^{2} a^{3}(0) \widetilde{w}_{1}^{\prime}(0) \widetilde{w}_{1,9}(0)-\lambda^{2} a^{3}(0) \widetilde{w}_{1, \vartheta}^{\prime}(0) \widetilde{w}_{1}(0)=-\int_{p_{0}}^{0} a(p) \widetilde{w}_{1}^{2}(p) \mathrm{d} p
$$

The partial derivative $W_{\vartheta}(0 ; \lambda, \vartheta)$ is given by

$$
W_{\vartheta}(0 ; \lambda, \vartheta)=\sigma \widetilde{w}_{1}(0)+\left(\lambda^{2} g \bar{\rho}(0)+\sigma \vartheta\right) \widetilde{w}_{1, \vartheta}(0)-\lambda^{2} a^{3}(0) \widetilde{w}_{1, \vartheta}^{\prime}(0) .
$$

Multiplying this equation by $\widetilde{w}_{1}(0)$ and using (4.37) and the colinearity of $\widetilde{w}_{1}$ and $\widetilde{w}_{2}$ we conclude that

$$
\widetilde{w}_{1}(0) W_{\vartheta}(0 ; \lambda, \vartheta)=\sigma \widetilde{w}_{1}^{2}(0)-\int_{p_{0}}^{0} a(p) \widetilde{w}_{1}^{2}(p) \mathrm{d} p .
$$

Next, we multiply $(4.16)_{1}$ by $\widetilde{w}_{1}$, integrate from $p_{0}$ to 0 , and use once more the fact that $\widetilde{w}_{1}$ and $\widetilde{w}_{2}$ are linearly dependent to obtain

$$
\sigma \widetilde{w}_{1}^{2}(0)-\int_{p_{0}}^{0} a(p) \widetilde{w}_{1}^{2}(p) \mathrm{d} p=-\frac{\lambda^{2}}{\vartheta}\left(g \bar{\rho}(0) \widetilde{w}_{1}^{2}(0)-\int_{p_{0}}^{0} a^{3}(p)\left(\widetilde{w}_{1}^{\prime}(p)\right)^{2} \mathrm{~d} p-\int_{p_{0}}^{0} g \bar{\rho}^{\prime}(p) \widetilde{w}_{1}^{2}(p) \mathrm{d} p .\right)
$$


Hence, (4.35) implies that

$$
W_{\vartheta}(0 ; \lambda, \vartheta)=-\frac{\lambda}{2 \vartheta} W_{\lambda}(0 ; \lambda, \vartheta)
$$

which completes the proof.

Given $\lambda>0$, let $\vartheta(\lambda)$ denote the largest solution to $W(0 ; \lambda, \vartheta)=0$. In the following lemma we identify, using Lemma 4.8, the mapping

$$
[\lambda \mapsto \vartheta(\lambda)]:(0, \infty) \rightarrow(0, \infty)
$$

up to a positive multiplicative constant.

Lemma 4.9 There exists a constant $C_{D}>0$ such that $\vartheta(\lambda)=C_{D} \lambda^{2}, \lambda>0$.

Proof Lemmas 4.7 and 4.8 ensure that $W_{\vartheta}(0 ; \lambda, \vartheta(\lambda))>0$ for all $\lambda>0$. The implicit function theorem applied at $\left(\lambda_{0}, \vartheta\left(\lambda_{0}\right)\right)$, with $\lambda_{0}>0$, implies that in a small neighborhood of $\left(\lambda_{0}, \vartheta\left(\lambda_{0}\right)\right)$ the solution set to $W(0 ; \lambda, \vartheta)=0$ coincides with the graph of a smooth curve $\widetilde{\vartheta}:\left(\lambda_{0}-\varepsilon, \lambda_{0}+\varepsilon\right) \rightarrow \mathbb{R}$. Differentiating the relation $W(0 ; \lambda, \widetilde{\vartheta}(\lambda))=0$ it follows in virtue of (4.38) that

$$
\widetilde{\vartheta}^{\prime}(\lambda)=-\frac{W_{\lambda}(0 ; \lambda, \widetilde{\vartheta}(\lambda))}{W_{\vartheta}(0 ; \lambda, \widetilde{\vartheta}(\lambda))}=\frac{2 \widetilde{\vartheta}(\lambda)}{\lambda} .
$$

Hence there exists a constant $C_{D}>0$ such that $\widetilde{\vartheta}(\lambda)=C_{D} \lambda^{2}$ for all $\lambda \in\left(\lambda_{0}-\varepsilon, \lambda_{0}+\varepsilon\right)$. The desired claim follows now at once.

Remark 4.10 Our analysis shows, under the assumptions (3.10) and (4.12), that the constant $C_{D}$ found in Lemma 4.9 is the largest constant such that the solutions to

$$
\left(a^{3} w^{\prime}\right)^{\prime}-g \bar{\rho}^{\prime} w-C_{D} a w=0 \quad \text { in } L_{r}\left(\left(p_{0}, 0\right)\right)
$$

determined by the initial data

$$
\left(w, w^{\prime}\right)\left(p_{0}\right)=(0,1) \quad \text { or } \quad\left(w, w^{\prime}\right)(0)=\left(a^{3}(0), g \bar{\rho}(0)+\sigma C_{D}\right) \text {, respectively, }
$$

are linearly dependent. The constant $C_{D}$ depends only on Earth's gravity $g$, the mass flux $p_{0}$, the water depth $d$, the surface tension coefficient $\sigma$, the density function $\bar{\rho}$, and on Bernoulli's function $\beta$. In the homogeneous case $\bar{\rho}=\rho \in \mathbb{R}$ we obtain, under the assumption that the flow is irrotational (that is for $\beta=0$ ), that $C_{D}$ is the largest positive solution to

$$
\tanh \left(d \sqrt{C_{D}}\right)=\frac{p_{0}^{2} \sqrt{C_{D}}}{d^{2}\left(g \rho+\sigma C_{D}\right)} .
$$

We cannot exclude the possibility that there exist finitely many (since $W(0 ; \lambda, \cdot): \mathbb{R} \rightarrow \mathbb{R}$ is real-analytic there cannot exist infinitely many) positive constants

$$
C_{D, N}<C_{D, N-1}<\cdots C_{D, 1}<C_{D}, \quad N \geq 1,
$$

for which the two solutions defined above are linearly dependent. Each of these constants defines a new function $\vartheta_{i}:(0, \infty) \rightarrow \mathbb{R}$ with 


$$
\vartheta_{i}(\lambda)=C_{D, i} \lambda^{2}, \quad 1 \leq i \leq N,
$$

which satisfies $W\left(0 ; \lambda, \vartheta_{i}(\lambda)\right)=0$ for all $\lambda>0$. This complicates the bifurcation analysis for (4.1) a lot as in this situation the dimension of $\operatorname{ker} \partial_{h} \mathcal{F}(\lambda, 0)$ may be larger than 1 for certain $\lambda$. However, this behavior is expected since, even in the case of constant density, phenomena like bifurcation from double eigenvalues or secondary bifurcation may occur when allowing for surface tension effects, cf. [35, 53, 54].

We now shortly discuss the dispersion relation (4.40) in the homogeneous irrotational case (that is for $\bar{\rho}=\rho \in \mathbb{R}$ and $\beta=0$ ). Setting $\sqrt{C_{D}}=x$, the problem reduces to finding the positive zeros of the function $f: \mathbb{R} \rightarrow \mathbb{R}$ with

$$
f(x):=\left(g \rho+\sigma x^{2}\right) \frac{\tanh (d x)}{x}-\frac{p_{0}^{2}}{d^{2}} .
$$

Since $f(0)=g \rho d-p_{0}^{2} / d^{2}<0$ (this inequality is a direct consequence of (4.12)) and $f(x) \rightarrow \infty$ as $x \rightarrow \infty$, Eq. (4.41) has at least a positive solution. Note that $f$ is even. Furthermore, direct computations show that

$$
f^{\prime \prime}(0)=2 d\left(\sigma-\frac{g \rho d^{2}}{3}\right) \text { and } f^{(4)}(0)=-8 d^{3}\left(\sigma-\frac{2 g \rho d^{2}}{5}\right) .
$$

Moreover, it can be shown that if $f^{\prime}(x)=0$ for some $x>0$, then $f^{\prime \prime}(x)>0$ (see, e.g., [38, Lemma 3]). Consequently, regardless of the sign of

$$
\sigma-\frac{g \rho d^{2}}{3}
$$

the equation $f(x)=0$ has a unique solution. Indeed, if $\sigma>g \rho d^{2} / 3$, then $f$ is strictly increasing on $(0, \infty)$. If $\sigma=g \rho d^{2} / 3$ then $f$ is again strictly increasing on $(0, \infty)$ since then $f^{(4)}(0)>0$. Finally, if $\sigma<g \rho d^{2} / 3$, then $f$ has a unique global minimizer let's say at $x_{0}$ and $f$ is strictly decreasing on $\left(0, x_{0}\right)$ and strictly increasing on $\left(x_{0}, \infty\right)$.

We proceed with the following result.

Proposition 4.11 Let

$$
\lambda_{*}:=\frac{2 \pi}{\sqrt{C_{D}}} .
$$

Then, $\partial_{h} \mathcal{F}\left(\lambda_{*}, 0\right)$ is a Fredholm operator of index zero and with a one-dimensional kernel spanned by

$$
w_{*}(q, p):=\widetilde{w}_{1}\left(p ; \lambda_{*},(2 \pi)^{2}\right) \cos (2 \pi q), \quad(p, q) \in \Omega,
$$

where $\widetilde{w}_{1}\left(\cdot ; \lambda_{*},(2 \pi)^{2}\right)$ denotes the solution to (4.16) corresponding to $(\lambda, \vartheta)=\left(\lambda_{*},(2 \pi)^{2}\right)$.

Proof The proof follows from the results established in Lemmas 4.1, 4.2, 4.4, 4.9, and Remark 4.10.

The transversality condition In order to apply [20, Theorem 1.7] to the bifurcation problem (4.1), we still have to check the transversality condition 


$$
\partial_{\lambda h} \mathcal{F}\left(\lambda_{*}, 0\right)\left[w_{*}\right] \notin \operatorname{Im} \partial_{h} \mathcal{F}\left(\lambda_{*}, 0\right),
$$

with $\lambda_{*}$ and $w_{*}$ introduced in (4.42) and (4.43), respectively. To this end we first characterize the set $\operatorname{Im} \partial_{h} \mathcal{F}\left(\lambda_{*}, 0\right)$.

Lemma 4.12 A pair $(f, \phi) \in \mathbb{Y}$ with $f=\varphi+\partial_{q} \psi_{1}+\partial_{p} \psi_{2}$ belongs to $\operatorname{Im} \partial_{h} \mathcal{F}\left(\lambda_{*}, 0\right)$ if and only if

$$
\int_{\Omega}\left(\psi_{1} w_{*, q}+\psi_{2} w_{*, p}-\varphi w_{*}\right) \mathrm{d}(q, p)-\int_{0}^{1} \operatorname{tr}_{0}\left(\psi_{2} w_{*}\right) d q-\sigma\left(1+(2 \pi)^{2}\right) \int_{0}^{1} \phi \operatorname{tr}_{0} w_{*} \mathrm{~d} q=0 .
$$

Proof Let $(f, \phi) \in \operatorname{Im} \partial_{h} \mathcal{F}\left(\lambda_{*}, 0\right)$ and let $w \in \mathbb{X}$ satisfy $(L, T)[w]=(f, \phi)$. Testing the equation $L[w]=f$ with $\xi_{m} w_{*} \in H_{0}^{1}(\Omega)$, where $w_{*}$ is defined in (4.43) and with $\xi_{m}$ defined by

$$
\xi_{m}(p):= \begin{cases}m\left(p-p_{0}\right), & p_{0} \leq p \leq p_{0}+1 / m, \\ 1, & p_{0}+1 / m \leq p \leq-1 / m, \quad p \in\left[p_{0}, 0\right], \frac{2}{\left|p_{0}\right|} \leq m \in \mathbb{N}, \\ -m p, & -1 / m \leq p \leq 0,\end{cases}
$$

we obtain, after passing to the limit $m \rightarrow \infty$, the following identity

$$
\begin{gathered}
\int_{\Omega}\left(\frac{w_{q} w_{*, q}}{H^{\prime}}+\lambda^{2} \frac{w_{p} w_{*, p}}{H^{\prime 3}}+\lambda^{2} g \bar{\rho}^{\prime} w w_{*}\right) \mathrm{d}(q, p)-\lambda^{2} \int_{0}^{1} \operatorname{tr}_{0} \frac{w_{p} w_{*}}{H^{\prime 3}} \mathrm{~d} q \\
=\int_{\Omega}\left(\psi_{1} w_{*, q}+\psi_{2} w_{*, p}-\varphi w_{*}\right) \mathrm{d}(q, p)-\int_{0}^{1} \operatorname{tr}_{0}\left(\psi_{2} w_{*}\right) \mathrm{d} q .
\end{gathered}
$$

Moreover, multiplying the relation $T[w]=\phi$ by $\operatorname{tr}_{0} w_{*}$ and integrating over $[0,1]$, it follows that

$$
\int_{0}^{1} \operatorname{tr}_{0}\left[\left(\sigma(2 \pi)^{2}+\lambda^{2} g \bar{\rho}\right) w w_{*}\right] \mathrm{d} q-\lambda^{2} \int_{0}^{1} \operatorname{tr}_{0} \frac{w_{p} w_{*}}{H^{3}} \mathrm{~d} q=\sigma\left(1+(2 \pi)^{2}\right) \int_{0}^{1} \phi \operatorname{tr}_{0} w_{*} \mathrm{~d} q
$$

Combining the relations (4.46) and (4.47) yields

$$
\begin{aligned}
& \int_{\Omega}\left(\frac{w_{q} w_{*, q}}{H^{\prime}}+\lambda^{2} \frac{w_{p} w_{*, p}}{H^{\prime 3}}+\lambda^{2} g \bar{\rho}^{\prime} w w_{*}\right) \mathrm{d}(q, p)-\int_{0}^{1} \operatorname{tr}_{0}\left[\left(\sigma(2 \pi)^{2}+\lambda^{2} g \bar{\rho}\right) w w_{*}\right] \mathrm{d} q \\
&=\int_{\Omega}\left(\psi_{1} w_{*, q}+\psi_{2} w_{*, p}-\varphi w_{*}\right) \mathrm{d}(q, p)-\int_{0}^{1} \operatorname{tr}_{0}\left(\psi_{2} w_{*}\right) \mathrm{d} q-\sigma\left(1+(2 \pi)^{2}\right) \int_{0}^{1} \phi \operatorname{tr}_{0} w_{*} \mathrm{~d} q .
\end{aligned}
$$

Finally, multiplying $L\left[w_{*}\right]=0$ by $w$ and integrating by parts, we find in virtue of

$$
\operatorname{tr}_{0} w_{*, p}=\operatorname{tr}_{0}\left[\left(\sigma(2 \pi)^{2}+\lambda^{2} g \bar{\rho}\right) \frac{H^{\prime 3}}{\lambda^{2}} w_{*}\right]
$$

cf. (4.43), that the left-hand side of (4.48) is zero and (4.45) follows. Observing that (4.45) defines a closed subspace of $\mathbb{Y}$ of codimension 1 which contains $\operatorname{Im} \partial_{h} \mathcal{F}\left(\lambda_{*}, 0\right)$, Proposition 4.11 leads us to the desired conclusion.

We are now in a position to prove that the transversality condition (4.44) holds. 
Proposition 4.13 Let $\lambda_{*}$ and $w_{*}$ be as defined in Proposition 4.11. It then holds

$$
\partial_{\lambda h} \mathcal{F}\left(\lambda_{*}, 0\right)\left[w_{*}\right] \notin \operatorname{Im} \partial_{h} \mathcal{F}\left(\lambda_{*}, 0\right) .
$$

Proof We compute that

$$
\partial_{\lambda h} \mathcal{F}\left(\lambda_{*}, 0\right)\left[w_{*}\right]=2 \lambda_{*}\left(\varphi+\partial_{p} \psi, \phi\right),
$$

where

$$
\varphi=g \bar{\rho} w_{*, p}, \quad \psi=\frac{w_{*, p}}{H^{\prime 3}}-g \bar{\rho} w_{*}, \quad \phi=-\frac{1}{\sigma\left(1+(2 \pi)^{2}\right)} \operatorname{tr}_{0} \psi
$$

Hence, according to Lemma 4.12, $\partial_{\lambda h} \mathcal{F}\left(\lambda_{*}, 0\right)\left[w_{*}\right] \in \operatorname{Im} \partial_{h} \mathcal{F}\left(\lambda_{*}, 0\right)$ if and only if

$$
\int_{\Omega}\left(\psi w_{*, p}-\varphi w_{*}\right) \mathrm{d}(q, p)-\int_{0}^{1} \operatorname{tr}_{0}\left(\psi w_{*}\right) \mathrm{d} q-\sigma\left(1+(2 \pi)^{2}\right) \int_{0}^{1} \phi \operatorname{tr}_{0} w_{*} \mathrm{~d} q=0 .
$$

The left-hand side of this equation can be expressed as

$$
\int_{\Omega}\left(\frac{w_{*, p}^{2}}{H^{\prime 3}}-g \bar{\rho}\left(w_{*}^{2}\right)_{p}\right) \mathrm{d}(q, p)=\frac{1}{2} \int_{p_{0}}^{0}\left(\frac{\left(\widetilde{w}_{1}^{\prime}\right)^{2}}{H^{\prime 3}}-g \bar{\rho}\left(\widetilde{w}_{1}^{2}\right)^{\prime}\right) \mathrm{d} p=-\frac{\widetilde{w}_{1}(0)}{4 \lambda_{*}} W_{\lambda}\left(0 ; \lambda_{*},(2 \pi)^{2}\right),
$$

which is positive according to Lemma 4.8. Thus, the assertion follows.

Improved regularity and the proof of Theorem 2.3 With this preparation completed we may now apply the bifurcation result [20, Theorem 1.7] to (4.1). This provides us with a local branch of weak solutions to (3.3) and (2.11) which contains, apart from the laminar flow defined by $\left(\lambda_{*}, 0\right)$ only nonlaminar solutions which belong to $\mathbb{X}$. We next prove that any weak solution $h \in \mathrm{C}^{1+\alpha}(\bar{\Omega})$ to (3.3) and (2.11), corresponding to some $\lambda>0$, is in fact a strong solution as defined in Theorem (2.1)(iii).

To this end we need the following regularity result.

Theorem 4.14 Let $\lambda>0, r \in(1, \infty), \alpha=(r-1) / r, \beta \in L_{r}\left(\left(p_{0}, 0\right)\right)$ and $\bar{\rho} \in W_{r}^{1}\left(\left(p_{0}, 0\right)\right)$. Given a weak solution $h \in \mathrm{C}^{1+\alpha}(\bar{\Omega})$ to $(3.3)$ and $(2.11)$, it holds that $\partial_{q}^{m} h \in \mathrm{C}^{1+\alpha}(\bar{\Omega})$ for all $m \in \mathbb{N}$ and there exists a constant $L>0$ such that

$$
\left\|\partial_{q}^{m} h\right\|_{C^{1+\alpha}(\bar{\Omega})} \leq L^{m-2}(m-3) ! \quad \text { for all } m \geq 3 .
$$

The proof of Theorem 4.14 is quite technical, but very similar to that of [44, Theorem 5.1] and is therefore omitted.

Remark 4.15 The estimate (4.49) implies in particular that all the streamlines, including the wave surface, of the corresponding strong solution to (3.3) and (2.11), see Proposition 4.17 and Theorem 2.1, are real-analytic graphs. Similar results for classical solutions to (3.3) and $(2.11)$ have been obtained in $[33,55]$ under the more restrictive assumption that $\bar{\rho}^{\prime}$, $\beta \in \mathrm{C}^{\alpha}\left(\left[p_{0}, 0\right]\right)$. We point out that the study of the a priori regularity of homogeneous but rotational waves has been initiated in [13], see also [26].

The next lemma plays an important role in the proof of Theorem 2.3(iii). 
Lemma 4.16 Given two solutions $\left(\lambda_{i}, h_{i}\right) \in(0, \infty) \times \mathbb{X}, i=1,2$, to (4.1), there exists a constant $C=C\left(\lambda_{1}, \lambda_{2},\left\|h_{1}\right\|_{\mathrm{C}^{1+\alpha}(\bar{\Omega})},\left\|h_{2}\right\|_{\mathrm{C}^{1+\alpha}(\bar{\Omega})},\left\|\partial_{q} h_{2}\right\|_{\mathrm{C}^{1+\alpha}(\bar{\Omega})}\right)$ such that

$$
\left\|\partial_{q} h_{1}-\partial_{q} h_{2}\right\|_{C^{1+\alpha}(\bar{\Omega})} \leq C\left(\left|\lambda_{1}-\lambda_{2}\right|+\left\|h_{1}-h_{2}\right\|_{C^{1+\alpha}(\bar{\Omega})}\right) .
$$

Proof Since $w_{i}:=\partial_{q} h_{i} \in \mathrm{C}^{1+\alpha}(\bar{\Omega})$, cf. Theorem 4.14, differentiation of the relations in Definition 3.1 shows that $w_{i}$ is a weak solution to the uniformly elliptic boundary value problem

$$
\begin{cases}\left(a_{i, 11} w_{i, q}\right)_{q}+\left(a_{i, 21} w_{i, p}\right)_{q}+\left(a_{i, 12} w_{i, q}\right)_{p}+\left(a_{i, 22} w_{i, p}+b_{i} w_{i}\right)_{p}+c_{i} w_{i, p}=0 & \text { in } \Omega, \\ w_{i}-\left(1-\partial_{q}^{2}\right)^{-1} \operatorname{tr}_{0}\left(A_{i} w_{i}+B_{i} w_{i, q}+C_{i} w_{i, p}\right)=0 & \text { on } p=0 \\ w_{i}=0 & \text { on } p=p_{0},\end{cases}
$$

where $a_{i, k \ell}, b_{i}, c_{i}, A_{i}, B_{i}, C_{i} \in \mathrm{C}^{\alpha}(\bar{\Omega})$ are given by

$$
\begin{aligned}
& a_{i, 11}=\frac{1}{h_{i, p}}, \quad a_{i, 12}=a_{i, 21}=-\frac{h_{i, q}}{h_{i, p}^{2}}, \quad a_{i, 22}=\frac{\lambda_{i}^{2}+h_{i, q}^{2}}{h_{i, p}^{3}}, \quad b_{i}=-\lambda_{i}^{2} g \bar{\rho}, \quad c_{i}=\lambda_{i}^{2} g \bar{\rho}, \\
& A_{i}=1-g \bar{\rho} \frac{\left(\lambda_{i}^{2}+h_{i, q}^{2}\right)^{3 / 2}}{\sigma \lambda_{i}}, \quad C_{i}=\frac{\left(\lambda_{i}^{2}+h_{i, q}^{2}\right)^{5 / 2}}{\sigma \lambda_{i}^{3} h_{i, p}^{3}}, \\
& B_{i}=-\frac{3\left(\lambda_{i}^{2}+h_{i, q}^{2}\right)^{1 / 2} h_{i, q}}{2 \sigma \lambda_{i}^{3}}\left(\frac{\lambda_{i}^{2}+h_{i, q}^{2}}{h_{i, p}^{2}}+2 \lambda_{i}^{2} g \bar{\rho}\left(h_{i}-d\right)-\int_{0}^{1} \frac{\lambda_{i}^{2}+h_{i, q}^{2}}{h_{i, p}^{2}} \mathrm{~d} q\right)-\frac{\left(\lambda_{i}^{2}+h_{i, q}^{2}\right)^{3 / 2} h_{i, q}}{\sigma \lambda_{i}^{3} h_{i, p}^{2}} .
\end{aligned}
$$

Hence, for the difference $W:=w_{1}-w_{2}$, we find that

$$
\begin{cases}\left(a_{1,11} W_{q}\right)_{q}+\left(a_{1,21} W_{p}\right)_{q}+\left(a_{1,12} W_{q}\right)_{p}+\left(a_{1,22} W_{p}+b_{i} W\right)_{p}+c_{1} W_{p}=f & \text { in } \Omega, \\ W=\varphi & \text { on } p=0 \\ W=0 & \text { on } p=p_{0}\end{cases}
$$

where

$$
\begin{aligned}
f= & \left(\left(a_{2,11}-a_{1,11}\right) w_{2, q}\right)_{q}+\left(\left(a_{2,21}-a_{1,21}\right) w_{2, p}\right)_{q}+\left(\left(a_{2,12}-a_{1,12}\right) w_{2, q}\right)_{p} \\
& +\left(\left(a_{2,22}-a_{1,22}\right) w_{2, p}+\left(b_{2}-b_{1}\right) w_{2}\right)_{p}+\left(c_{2}-c_{1}\right) w_{2, p}, \\
\varphi= & \left(1-\partial_{q}^{2}\right)^{-1} \operatorname{tr}_{0}\left(A_{1} W+B_{1} W_{q}+C_{1} W_{p}+w_{2}\left(A_{1}-A_{2}\right)+w_{2, q}\left(B_{1}-B_{2}\right)+w_{2, p}\left(C_{1}-C_{2}\right)\right) .
\end{aligned}
$$

Using [28, Theorem 8.33], we estimate

$$
\begin{aligned}
\|W\|_{C^{1+\alpha}(\bar{\Omega})} \leq & C\left(\|W\|_{C(\bar{\Omega})}+\|\varphi\|_{C^{1+\alpha}(\mathbb{R})}+\left\|\left(c_{2}-c_{1}\right) w_{2, p}\right\|_{C(\bar{\Omega})}+\left\|\left(a_{2,11}-a_{1,11}\right) w_{2, q}\right\|_{C^{\alpha}(\bar{\Omega})}\right. \\
& \left.+\left\|\left(a_{2,21}-a_{1,21}\right) w_{2, p}\right\|_{C^{\alpha}(\bar{\Omega})}+\left\|\left(a_{2,12}-a_{1,12}\right) w_{2, q}\right\|_{C^{\alpha}(\bar{\Omega})}\right) \\
& \left.+\left\|\left(a_{2,22}-a_{1,22}\right) w_{2, p}+\left(b_{2}-b_{1}\right) w_{2}\right\|_{C^{\alpha}(\bar{\Omega})}\right) \\
\leq & C\left(\|W\|_{C^{1+\alpha / 2}(\bar{\Omega})}+\|\varphi\|_{C^{1+\alpha}(\mathbb{R})}+\left|\lambda_{1}-\lambda_{2}\right|+\left\|h_{2}-h_{1}\right\|_{C^{1+\alpha}(\bar{\Omega})}\right)
\end{aligned}
$$

where, in virtue of $\left(1-\partial_{q}^{2}\right)^{-1} \in \mathcal{L}\left(\mathrm{C}^{\alpha / 2}(\mathbb{R}), \mathrm{C}^{2+\alpha / 2}(\mathbb{R})\right)$, it holds that 


$$
\begin{aligned}
\|\varphi\|_{\mathrm{C}^{1+\alpha}(\mathbb{R})} & \leq C\|\varphi\|_{\mathrm{C}^{2+\alpha / 2}(\mathbb{R})} \\
& \leq C\left\|A_{1} W+B_{1} W_{q}+C_{1} W_{p}+w_{2}\left(A_{1}-A_{2}\right)+w_{2, q}\left(B_{1}-B_{2}\right)+w_{2, p}\left(C_{1}-C_{2}\right)\right\|_{\mathrm{C}^{\alpha / 2}(\bar{\Omega})} \\
& \leq C\left(\|W\|_{\mathrm{C}^{1+\alpha / 2}(\bar{\Omega})}+\left|\lambda_{1}-\lambda_{2}\right|+\left\|h_{2}-h_{1}\right\|_{\mathrm{C}^{1+\alpha}(\bar{\Omega})}\right) .
\end{aligned}
$$

Hence

$$
\|W\|_{\mathrm{C}^{1+\alpha}(\bar{\Omega})} \leq C\left(\|W\|_{\mathrm{C}^{1+\alpha / 2}(\bar{\Omega})}+\left|\lambda_{1}-\lambda_{2}\right|+\left\|h_{2}-h_{1}\right\|_{\mathrm{C}^{1+\alpha}(\bar{\Omega})}\right),
$$

and the desired claim follows by the interpolation result $\mathrm{C}^{1+\alpha / 2}(\bar{\Omega})=\left(\mathrm{C}^{\alpha}(\bar{\Omega}), \mathrm{C}^{1+\alpha}(\bar{\Omega})\right)_{1-\alpha / 2, \infty}$.

We next prove that weak solutions to (3.3) and (2.11) are in fact strong solutions (as defined in Theorem 2.1 (iii)).

Proposition 4.17 Let $\lambda>0$ and let $h \in \mathrm{C}^{1+\alpha}(\bar{\Omega})$ denote a weak solution to (3.3) and (2.11). Then $h \in W_{r}^{2}(\Omega)$ is a strong solution to (3.3) and (2.11).

Proof In virtue of Theorem 4.14 we have $h_{q} \in \mathrm{C}^{1+\alpha}(\bar{\Omega})$, hence $\partial_{q} h_{p}=\partial_{p} h_{q} \in \mathrm{C}^{\alpha}(\bar{\Omega})$. This in turn implies that $\partial_{q} h_{p}$ is the classical derivative of $h_{p}$ with respect to $q$. Recalling that

$$
\left(\frac{h_{q}}{h_{p}}\right)_{q}-\left(\frac{\lambda^{2}+h_{q}^{2}}{2 h_{p}^{2}}+\lambda^{2} B+\lambda^{2} g \bar{\rho}(h-d)\right)_{p}+\lambda^{2} g \bar{\rho} h_{p}=0 \quad \text { in } \mathcal{D}^{\prime}(\Omega),
$$

it follows that

$$
\left(\frac{\lambda^{2}+h_{q}^{2}}{2 h_{p}^{2}}+\lambda^{2} B+\lambda^{2} g \bar{\rho}(h-d)\right)_{q} \in \mathrm{C}^{\alpha}(\bar{\Omega}), \quad\left(\frac{\lambda^{2}+h_{q}^{2}}{2 h_{p}^{2}}+\lambda^{2} B+\lambda^{2} g \bar{\rho}(h-d)\right)_{p} \in \mathrm{C}^{\alpha}(\bar{\Omega}),
$$

and (2.14) in turn yields

$$
\frac{\lambda^{2}+h_{q}^{2}}{2 h_{p}^{2}}+\lambda^{2} B+\lambda^{2} g \bar{\rho}(h-d) \in \mathrm{C}^{1+\alpha}(\bar{\Omega}) .
$$

Consequently,

$$
\frac{\lambda^{2}+h_{q}^{2}}{2 h_{p}^{2}} \in W_{r}^{1}(\Omega) \cap \mathrm{C}^{\alpha}(\bar{\Omega})
$$

and the repeated use of [28, Lemma 7.5] finally yields $h_{p} \in W_{r}^{1}(\Omega)$. Since $h_{q} \in \mathrm{C}^{1+\alpha}(\bar{\Omega})$, it

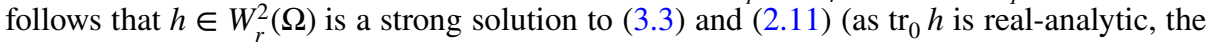
condition $\operatorname{tr}_{0} h \in W_{r}^{2}(\mathbb{R})$ is obvious).

We complete this section with the proof of Theorem 2.3.

Proof of Theorem 2.3 Gathering (4.2), (4.3), Propositions 4.11, and 4.13 we find that all assumptions of the theorem on bifurcation from simple eigenvalues by Crandall and 
Rabinowitz, cf. [20, Theorem 1.7], are satisfied in the context of the bifurcation problem (4.1). This abstract result yields the existence of a local smooth curve

$$
[s \mapsto(\lambda(s), h(s))]:(-\varepsilon, \varepsilon) \rightarrow(0, \infty) \times \mathbb{X},
$$

where $\varepsilon>0$ is in general a small number, such that $\mathcal{F}(\lambda(s), h(s))=0$ for all $|s|<\varepsilon$. Moreover, $\lambda(0)=\lambda_{*}$ and

$$
h(s)=s\left(w_{*}+\chi(s)\right)
$$

with $\chi \in \mathrm{C}^{\infty}((-\varepsilon, \varepsilon), \mathbb{X})$ satisfying $\chi(0)=0$. Besides, there exists a ball in $(0, \infty) \times \mathbb{X}$, with center $\left(\lambda_{*}, 0\right)$, which does not contain other solutions but those mentioned above or trivial solutions $(\lambda, 0)$. Letting $H$ denote the laminar flow solution fixed at the beginning of the section, it follows from Proposition 4.17 that $H+h(s)$ is a strong solution to (3.3) and (2.11) having minimal wavelength 1 for $s \neq 0$. Each pair $(\lambda(s), H+h(s))$ corresponds to a solution $(u(s)-c, v(s), P(s), \rho(s), \eta(s))$ to (2.2)-(2.3) which lies on the curve $\mathcal{C}$ in Theorem 2.3 and which has minimal period $\lambda(s)$ (provided that $s \neq 0$ ), cf. (3.2) and Theorem 2.1. This proves the claims (i), (ii), and (iv) of Theorem 2.3.

It remains to show that $\eta(s)$ has, for $s \neq 0$, precisely one maximum and one minimum in $[0, \lambda(s))$ and that $\eta(s)$ it is strictly monotone between the points where the global extrema are attained. To this end we claim that

$$
\partial_{q} \chi(s) \underset{s \rightarrow 0}{\rightarrow} 0 \text { in } \mathrm{C}^{1+\alpha / 2}(\bar{\Omega}) .
$$

Indeed, Lemma 4.16 and (4.43) imply that there exists a constant $C>0$ such that

$$
\left\|\partial_{q} \chi(s)\right\|_{\mathrm{C}^{1+\alpha}(\bar{\Omega})} \leq\left\|\frac{\partial_{q} h(s)-\partial_{q} h(0)}{s}\right\|_{\mathrm{C}^{1+\alpha}(\bar{\Omega})}+\left\|\partial_{q} w_{*}\right\|_{\mathrm{C}^{1+\alpha}(\bar{\Omega})} \leq C
$$

for all $|s|<\varepsilon / 2$. Additionally, using the differentiability of $h$ at $s=0$, we have

$$
\left\|\partial_{q} \chi(s)\right\|_{\mathrm{C}^{\alpha}(\bar{\Omega})}=\left\|\frac{\partial_{q} h(s)-\partial_{q} h(0)-s \partial_{q} h^{\prime}(0)}{s}\right\|_{\mathrm{C}^{\alpha}(\bar{\Omega})} \leq\left\|\frac{h(s)-h(0)-s h^{\prime}(0)}{s}\right\|_{\mathrm{C}^{1+\alpha}(\bar{\Omega})} \underset{s \rightarrow 0}{\rightarrow} 0 .
$$

These relations together with the interpolation result $C^{1+\alpha / 2}(\bar{\Omega})=\left(C^{\alpha}(\bar{\Omega}), C^{1+\alpha}(\bar{\Omega})\right)_{1-\alpha / 2, \infty}$ immediately yield (4.53). Since $h(s, \cdot, 0)=s\left(w_{*}(\cdot, 0)+\chi(s, \cdot, 0)\right)$ and $\chi(s, \cdot, 0) \rightarrow 0$ in $\mathrm{C}^{2}(\mathbb{R})$ for $s \rightarrow 0$, cf. (4.52)-(4.53), standard arguments show that the monotonicity properties of $w_{*}$ are inherited by $h(s, \cdot, 0)$ provided that $\varepsilon$ is sufficiently small (see, e.g., [50]). Recalling that the wave profile is parameterized by the function $\eta(s)=h(s, \cdot, 0)-d$, we have established (iii) and the proof is complete.

Acknowledgements Open Access funding provided by Projekt DEAL. Patrik Knopf and Bogdan-Vasile Matioc were partially supported by the RTG 2339 "Interfaces, Complex Structures, and Singular Limits" of the German Science Foundation (DFG). Christina Lienstromberg has been supported by the Deutsche Forschungsgemeinschaft (DFG, German Research Foundation) through the collaborative research centre 'The mathematics of emerging effects' (CRC 1060, Projekt-ID 211504053 ) and the Hausdorff Center for Mathematics (GZ 2047/1, Projekt-ID 390685813). The support is gratefully acknowledged.

Open Access This article is licensed under a Creative Commons Attribution 4.0 International License, which permits use, sharing, adaptation, distribution and reproduction in any medium or format, as long as you give appropriate credit to the original author(s) and the source, provide a link to the Creative Commons licence, and indicate if changes were made. The images or other third party material in this article are included in the 
article's Creative Commons licence, unless indicated otherwise in a credit line to the material. If material is not included in the article's Creative Commons licence and your intended use is not permitted by statutory regulation or exceeds the permitted use, you will need to obtain permission directly from the copyright holder. To view a copy of this licence, visit http://creativecommons.org/licenses/by/4.0/.

\section{References}

1. Alt, H.W.: Linear Functional Analysis. Universitext, Springer, London (An Application-Oriented Introduction, Translated from the German edition by Robert Nürnberg) (2016)

2. Amann, H.: Ordinary Differential Equations, vol. 13 of de Gruyter Studies in Mathematics, Walter de Gruyter \& Co., Berlin (1990) (An Introduction to Nonlinear Analysis, Translated from the German by Gerhard Metzen)

3. Ambrose, D.M., Strauss, W.A., Wright, J.D.: Global bifurcation theory for periodic traveling interfacial gravity-capillary waves. Ann. Inst. Henri Poincaré Anal. Non Linéaire 33, 1081-1101 (2016)

4. Amick, C.J., Turner, R.E.L.: Small internal waves in two-fluid systems. Arch. Ration. Mech. Anal. 108, 111-139 (1989)

5. Benjamin, B.T.: The solitary wave on a stream with an arbitrary distribution of vorticity. J. Fluid Mech. 12, 97-116 (1962)

6. Chen, R.M., Walsh, S.: Continuous dependence on the density for stratified steady water waves. Arch. Ration. Mech. Anal. 219, 741-792 (2016)

7. Chen, R.M., Walsh, S.: Unique determination of stratified steady water waves from pressure. J. Differ. Equ. 264, 115-133 (2018)

8. Chen, R.M., Walsh, S., Wheeler, M.H.: Existence and qualitative theory for stratified solitary water waves. Ann. Inst. Henri Poincaré Anal. Non Linéaire 35, 517-576 (2018)

9. Constantin, A.: Edge waves along a sloping beach. J. Phys. A 34, 9723 (2001)

10. Constantin, A.: On the deep water wave motion. J. Phys. A 34, 1405 (2001)

11. Constantin, A.: Nonlinear Water Waves with Applications to Wave-Current Interactions and Tsunamis, vol. 81 of CBMS-NSF Conference Series in Applied Mathematics. SIAM, Philadelphia (2011)

12. Constantin, A.: Some three-dimensional nonlinear equatorial flows. J. Phys. Oceanogr. 43, 165-175 (2013)

13. Constantin, A., Escher, J.: Analyticity of periodic traveling free surface water waves with vorticity. Ann. Math. 173, 559-568 (2011)

14. Constantin, A., Ivanov, R.I.: A Hamiltonian approach to wave-current interactions in two-layer fluids. Phys. Fluids 27, 086603 (2015)

15. Constantin, A., Ivanov, R.I., Martin, C.-I.: Hamiltonian formulation for wave-current interactions in stratified rotational flows. Arch. Ration. Mech. Anal. 221, 1417-1447 (2016)

16. Constantin, A., Johnson, R.S.: An exact solution for equatorially trapped waves. J. Geophys. Res. Oceans 117, C05029 (2012)

17. Constantin, A., Johnson, R.S.: An exact, steady, purely azimuthal equatorial flow with a free surface. J. Phys. Oceanogr. 46, 1935-1945 (2016)

18. Constantin, A., Strauss, W.: Exact steady periodic water waves with vorticity. Commun. Pure Appl. Math. 57, 481-527 (2004)

19. Constantin, A., Strauss, W.: Periodic traveling gravity water waves with discontinuous vorticity. Arch. Ration. Mech. Anal. 202, 133-175 (2011)

20. Crandall, M.G., Rabinowitz, P.H.: Bifurcation from simple eigenvalues. J. Funct. Anal. 8, 321-340 (1971)

21. Cushman-Roisin, B., Beckers, J.-M.: Introduction to Geophysical Fluid Dynamics. Academic Press, London (2009)

22. Dubreil-Jacotin, M.-L.: Sur les ondes de type permanent dans les liquides hétérogènes. Atti Accad. Naz. Lincei Rend. 6, 814-819 (1932)

23. Dubreil-Jacotin, M.-L.: Sur les théorèmes d'èxistence relatifs aux ondes permanentes périodiques a deux dimensions dans les liquides hétérogènes. J. Math. Pures. Appl. 9, 43-67 (1937)

24. Ehrnström, M., Wahlén, E.: Trimodal steady water waves. Arch. Ration. Mech. Anal. 216, 449-471 (2015)

25. Escher, J., Matioc, A.-V., Matioc, B.-V.: On stratified steady periodic water waves with linear density distribution and stagnation points. J. Differ. Equ. 251, 2932-2949 (2011)

26. Escher, J., Matioc, B.-V.: On the analyticity of periodic gravity water waves with integrable vorticity function. Differ. Integr. Equ. 27, 217-232 (2014) 
27. Gerstner, F.: Theorie der Wellen samt einer daraus abgeleiteten Theorie der Deichprofile. Ann. Phys. 2, 412-445 (1809)

28. Gilbarg, D., Trudinger, N.S.: Elliptic Partial Differential Equations of Second Order. Springer, Berlin (2001)

29. Henry, D., Martin, C.I.: Exact, purely azimuthal stratified equatorial flows in cylindrical coordinates. Dyn. Partial Differ. Equ. 15, 337-349 (2018)

30. Henry, D., Martin, C.I.: Exact, free-surface equatorial flows with general stratification in spherical coordinates. Arch. Ration. Mech. Anal. 233, 497-512 (2019)

31. Henry, D., Martin, C.I.: Free-surface, purely azimuthal equatorial flows in spherical coordinates with stratification. J. Differ. Equ. 266, 6788-6808 (2019)

32. Henry, D., Matioc, A.-V.: Global bifurcation of capillary-gravity stratified water waves. Proc. R. Soc. Edinb. Sect. A 144, 775-786 (2014)

33. Henry, D., Matioc, B.-V.: On the regularity of steady periodic stratified water waves. Commun. Pure Appl. Anal. 11, 1453-1464 (2012)

34. Henry, D., Matioc, B.-V.: On the existence of steady periodic capillary-gravity stratified water waves. Ann. Scuola Norm. Sup. Pisa XII, 955-974 (2013)

35. Jones, M.C.W.: Small amplitude capillary-gravity waves in a channel of finite depth. Glasg. Math. J. 31, 141-160 (1989)

36. Kluczek, M.: Exact Pollard-like internal water waves. J. Nonlinear Math. Phys. 26, 133-146 (2019)

37. Long, R.R.: Some aspects of the flow of stratified fluids. I. A theoretical investigation. Tellus 5, 42-58 (1953)

38. Martin, C.I.: Local bifurcation and regularity for steady periodic capillary-gravity water waves with constant vorticity. Nonlinear Anal. Real World Appl. 14, 131-149 (2013)

39. Martin, C.I.: Hamiltonian structure for rotational capillary waves in stratified flows. J. Differ. Equ. 261, 373-395 (2016)

40. Martin, C.I.: A Hamiltonian approach for nonlinear rotational capillary-gravity water waves in stratified flows. Discrete Contin. Dyn. Syst. 37, 387-404 (2017)

41. Martin, C.I., Matioc, B.-V.: Steady periodic water waves with unbounded vorticity: equivalent formulations and existence results. J. Nonlinear Sci. 24, 633-659 (2014)

42. Matioc, A.-V.: Steady internal water waves with a critical layer bounded by the wave surface. J. Nonlinear Math. Phys. 19, 1250008, 21 (2012)

43. Matioc, A.-V.: Exact geophysical waves in stratified fluids. Appl. Anal. 92, 2254-2261 (2013)

44. Matioc, A.-V., Matioc, B.-V.: Capillary-gravity water waves with discontinuous vorticity: existence and regularity results. Commun. Math. Phys. 330, 859-886 (2014)

45. Matioc, B.-V.: Global bifurcation for water waves with capillary effects and constant vorticity. Monatsh. Math. 174, 459-475 (2014)

46. Pedlosky, J.: Geophysical Fluid Dynamics. Springer, New York (1979)

47. Pinet, P.R.: Invitation to Oceanography. Jones \& Bartlett Publishers, Burlington (2013)

48. Stuhlmeier, R.: On edge waves in stratified water along a sloping beach. J. Nonlinear Math. Phys 18, 127-137 (2011)

49. Turner, R.E.L.: Internal waves in fluids with rapidly varying density. Ann. Scuola Norm.Sup. Pisa Cl. Sci. 4(8), 513-573 (1981)

50. Wahlén, E.: Steady periodic capillary-gravity waves with vorticity. SIAM J. Math. Anal. 38, 921-943 (2006). (electronic)

51. Walsh, S.: Some criteria for the symmetry of stratified water waves. Wave Motion 46, 350-362 (2009)

52. Walsh, S.: Stratified steady periodic water waves. SIAM J. Math. Anal. 41, 1054-1105 (2009)

53. Walsh, S.: Steady stratified periodic gravity waves with surface tension I: local bifurcation. Discrete Contin. Dyn. Syst. 34, 3241-3285 (2014)

54. Walsh, S.: Steady stratified periodic gravity waves with surface tension II: global bifurcation. Discrete Contin. Dyn. Syst. 34, 3287-3315 (2014)

55. Wang, L.-J.: Regularity of traveling periodic stratified water waves with vorticity. Nonlinear Anal. 81, 247-263 (2013)

56. Yih, C.-S.: Exact solutions for steady two-dimensional flow of a stratified fluid. J. Fluid Mech. 9, 161$174(1960)$

Publisher's Note Springer Nature remains neutral with regard to jurisdictional claims in published maps and institutional affiliations. 